\title{
Visible-light Promoted Selenocyanation of Cyclobutanone Oxime Esters using Potassium Selenocyanate
}

Xia Zhao, ${ }^{\dagger}, *$ Liangshuo $\mathrm{Ji}^{\dagger}{ }^{\dagger} \mathrm{Yu}$ gao,,$^{\dagger}$ Tengteng Sun $,{ }^{\dagger} \mathrm{Jiamin}_{\mathrm{Qiao}},^{\dagger}$ Ankun $\mathrm{Li}^{\dagger}$ and Kui Lu*

${ }^{\dagger}$ College of Chemistry, Tianjin Key Laboratory of Structure and Performance for Functional Molecules, Key laboratory of Inorganic-organic Hybrid Functional Material Chemistry, Ministry of Education, Tianjin Normal University, Tianjin, China, 300387

$\$$ China International Science and Technology Cooperation Base of Food Nutrition/Safety and Medicinal Chemistry, College of Biotechnology, Tianjin University of Science \& Technology, Tianjin, China, 300457

*E-mail: hxxyzhx@mail.tjnu.edu.cn;

\section{Contents}

Page

1) Detailed Optimization of Reaction Conditions S2-S4

2) NMR spectra $\quad$ S5-S39

3) X-ray crystallography structure of compound 6c. S41-S51 


\section{Detailed Optimization of Reaction Conditions}

\subsection{Optimization of Reaction Conditions}

Table S1. The effect of photocatalyst equivalent

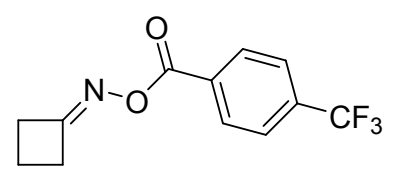

$1 a$

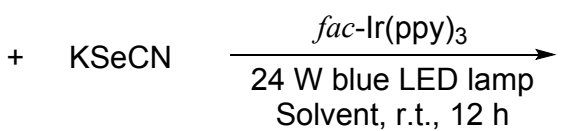

2
NC<smiles>CCC[Se]C#N</smiles>

$3 a$

\begin{tabular}{lll}
\hline Entry & Photocatalyst (mol \%) & Yield $(\%)$ \\
\hline 1 & $f a c-\operatorname{lr}(\mathrm{ppy})_{3}(0.8)$ & 77 \\
2 & $f a c-\operatorname{lr}(\mathrm{ppy})_{3}(1.0)$ & 85 \\
3 & $f a c-\operatorname{lr}(\mathrm{ppy})_{3}(1.2)$ & 83 \\
\hline
\end{tabular}

a Conditions: 1a $(0.5 \mathrm{mmol}), 2 \mathrm{a}(1.25 \mathrm{mmol})$, photocatalyst $(1 \mathrm{~mol} \%)$, solvent $(2 \mathrm{~mL}), 24 \mathrm{~W}$ blue LED lamp, r.t., $12 \mathrm{~h}$.

Table S2. The effect of concentrations

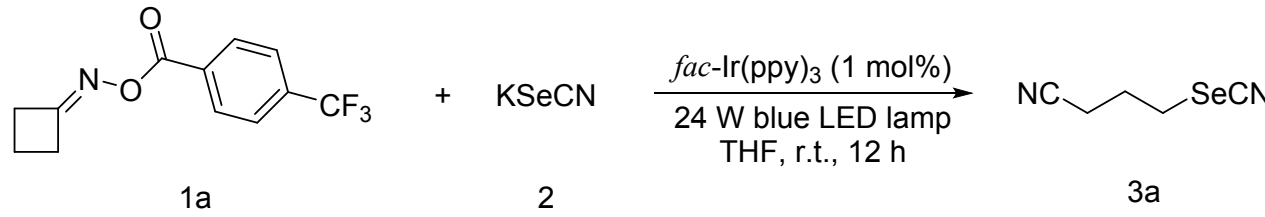

\begin{tabular}{lll}
\hline Entry & Concentration $(\mathrm{X} \mathrm{mL})$ & Yield (\%) \\
\hline 1 & $0.30 \mathrm{M}(1.5 \mathrm{~mL})$ & 77 \\
2 & $0.25 \mathrm{M}(2.0 \mathrm{~mL})$ & 85 \\
3 & $0.20 \mathrm{M}(2.5 \mathrm{~mL})$ & 83 \\
4 & $0.14 \mathrm{M}(3.5 \mathrm{~mL})$ & 83 \\
\hline
\end{tabular}

a The same with entry 1 in Table S1 with different amount of THF. 
Table S3. The effect of 2 a equivalent

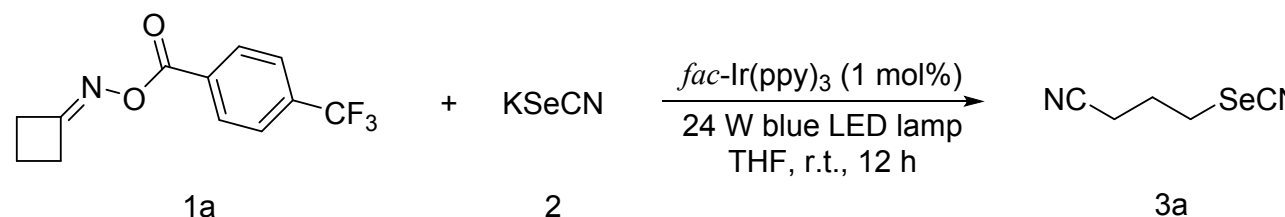

$1 a$

$23 a$

\begin{tabular}{lll}
\hline Entry & 2a eq. & Yield (\%) \\
\hline 1 & 1.5 & 79 \\
2 & 2.0 & 84 \\
3 & 2.5 & 92 \\
4 & 3.0 & 84 \\
\hline
\end{tabular}

a The same with entry 1 in Table S1 with different 2a equivalent.

Table S4. The effect of light source

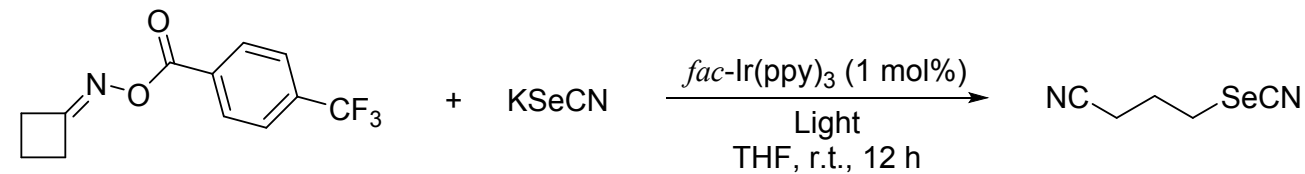

$1 a$

2

$3 a$

\begin{tabular}{lll}
\hline Entry & Light & Yield (\%) \\
\hline 1 & 24 W white LED lamp & trace \\
2 & 24 W green LED lamp & trace \\
3 & 18 W blue LED lamp & 90 \\
4 & 36 W blue LED lamp & 83 \\
\hline
\end{tabular}

a The same with entry 1 in Table S1 with different light. 
Table S5. Leaving Group<smiles>[R]c1ccc(C(=O)ON=C2CCC2)cc1</smiles><smiles>O=C(ON=C1CCC1)c1ccc(C(F)(F)F)cc1</smiles>

$1 \mathrm{a}$<smiles>O=C(ON=C1CCC1)c1c(F)c(F)c(F)c(F)c1F</smiles>

1a-1<smiles>O=C(ON=C1CCC1)c1ccccc1</smiles>

1a-2

\begin{tabular}{lll}
\hline Entry & 1 & Yield (\%) \\
\hline 1 & $1 \mathrm{a}$ & 92 \\
2 & $1 \mathrm{a}-1$ & trace \\
3 & $1 \mathrm{a}-2$ & N.R. \\
\hline
\end{tabular}

a The same with entry 1 in Table S1 with different leaving group.

\section{Table S6. Control experiments}

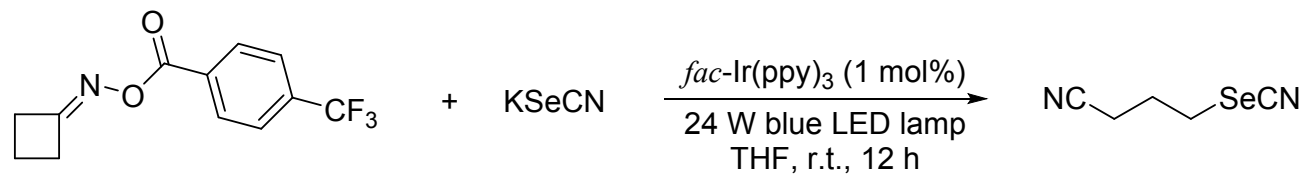

$1 \mathrm{a}$

2

$3 a$

\begin{tabular}{llll}
\hline Entry & hv & Photocatalyst & Yield (\%) \\
\hline 1 & + & - & N.R. \\
2 & - & + & N.R. \\
3 & + & + & 92 \\
$4^{\mathrm{b}}$ & + & + & 73 \\
\hline
\end{tabular}

a The same with entry 1 in Table $S 1 .^{b}$ in air.

LED lamp used in our experiment
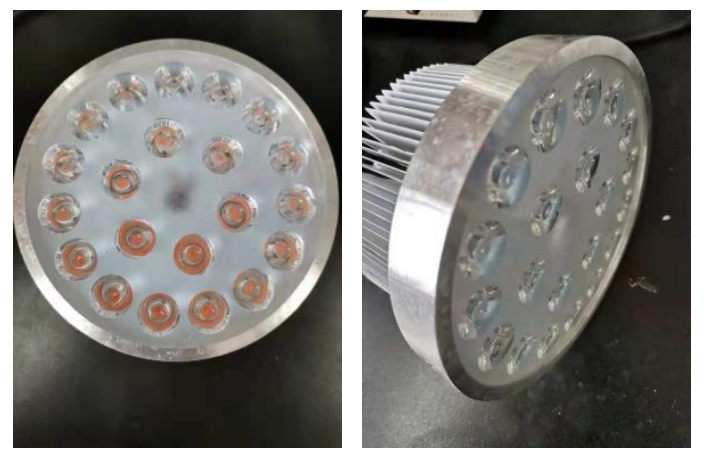


\section{Copies of ${ }^{1} \mathrm{H}$ and ${ }^{13} \mathrm{C}$ NMR Spectra for Substrates and Products}
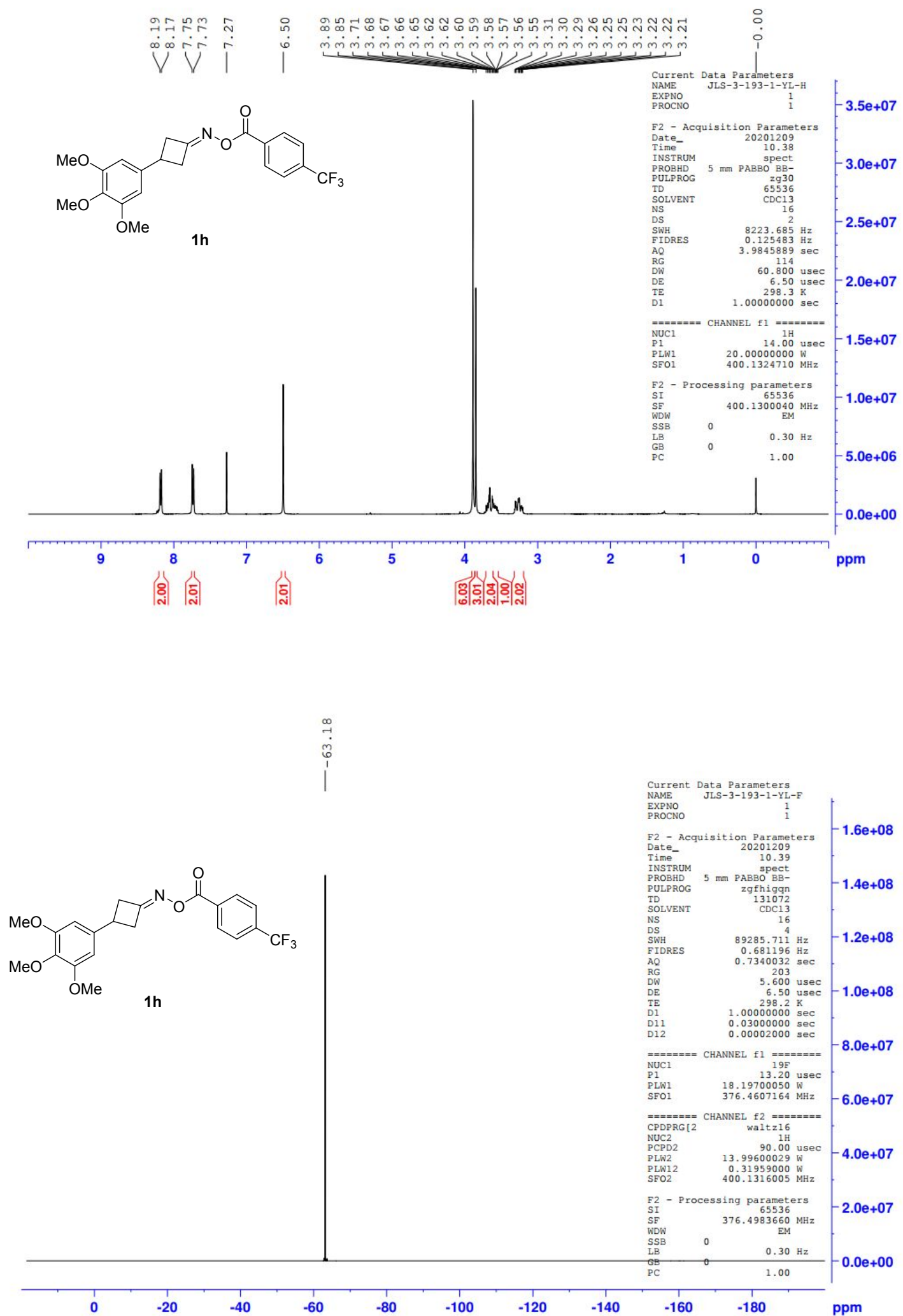

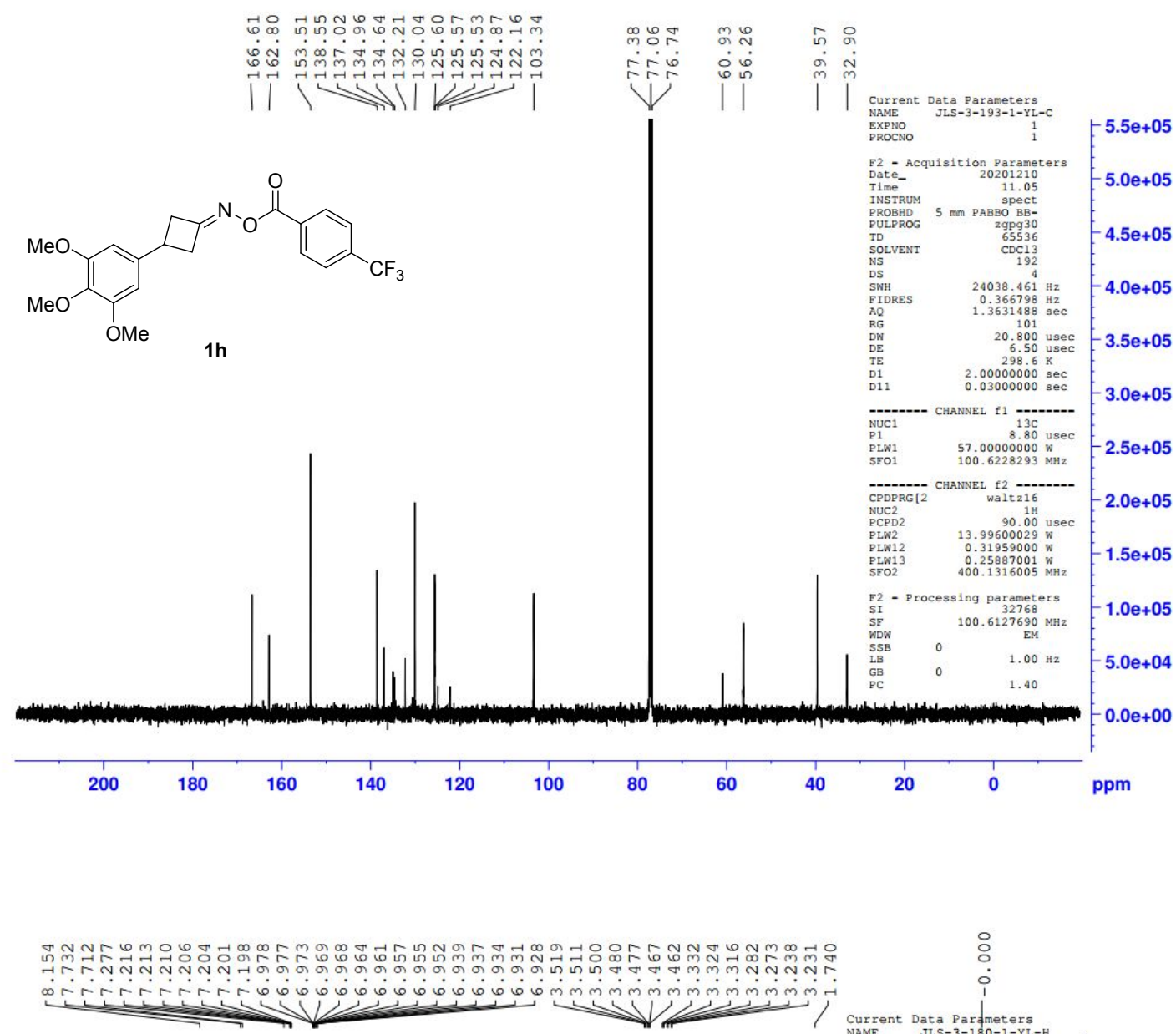

:
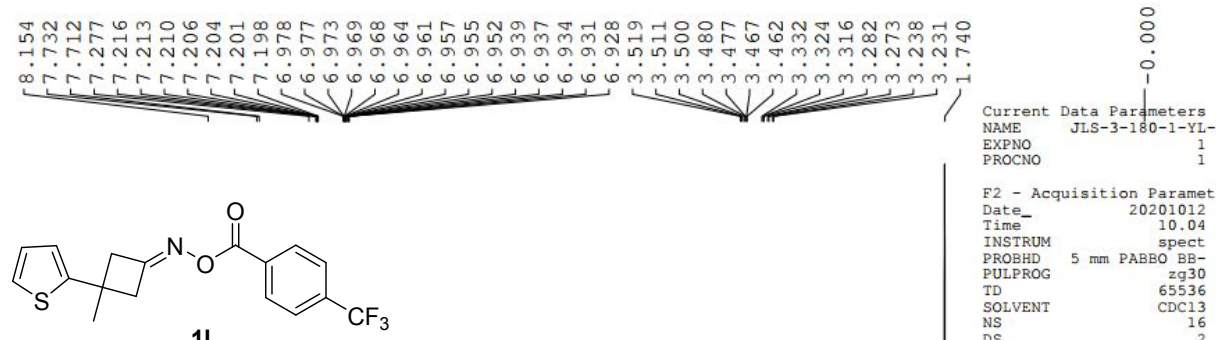

11

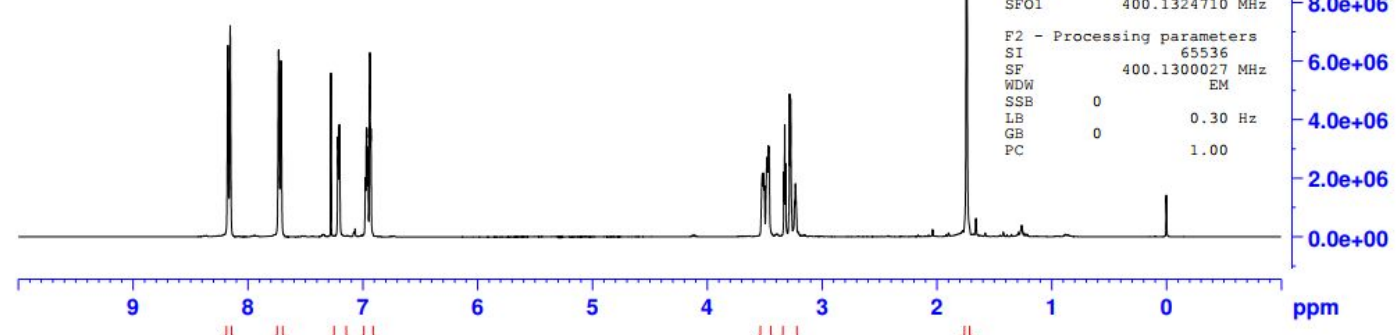

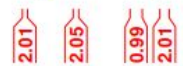

ไั

일 

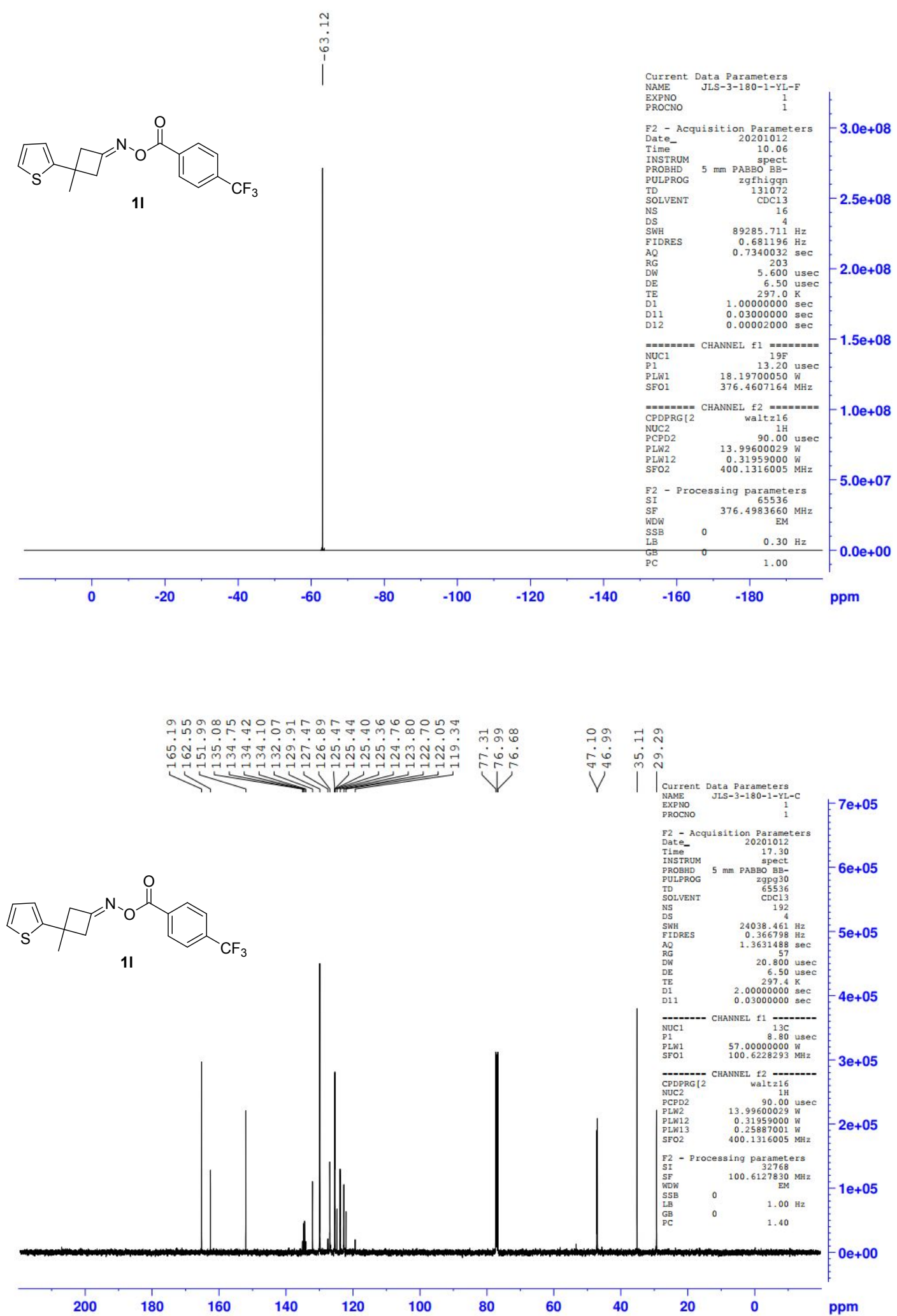

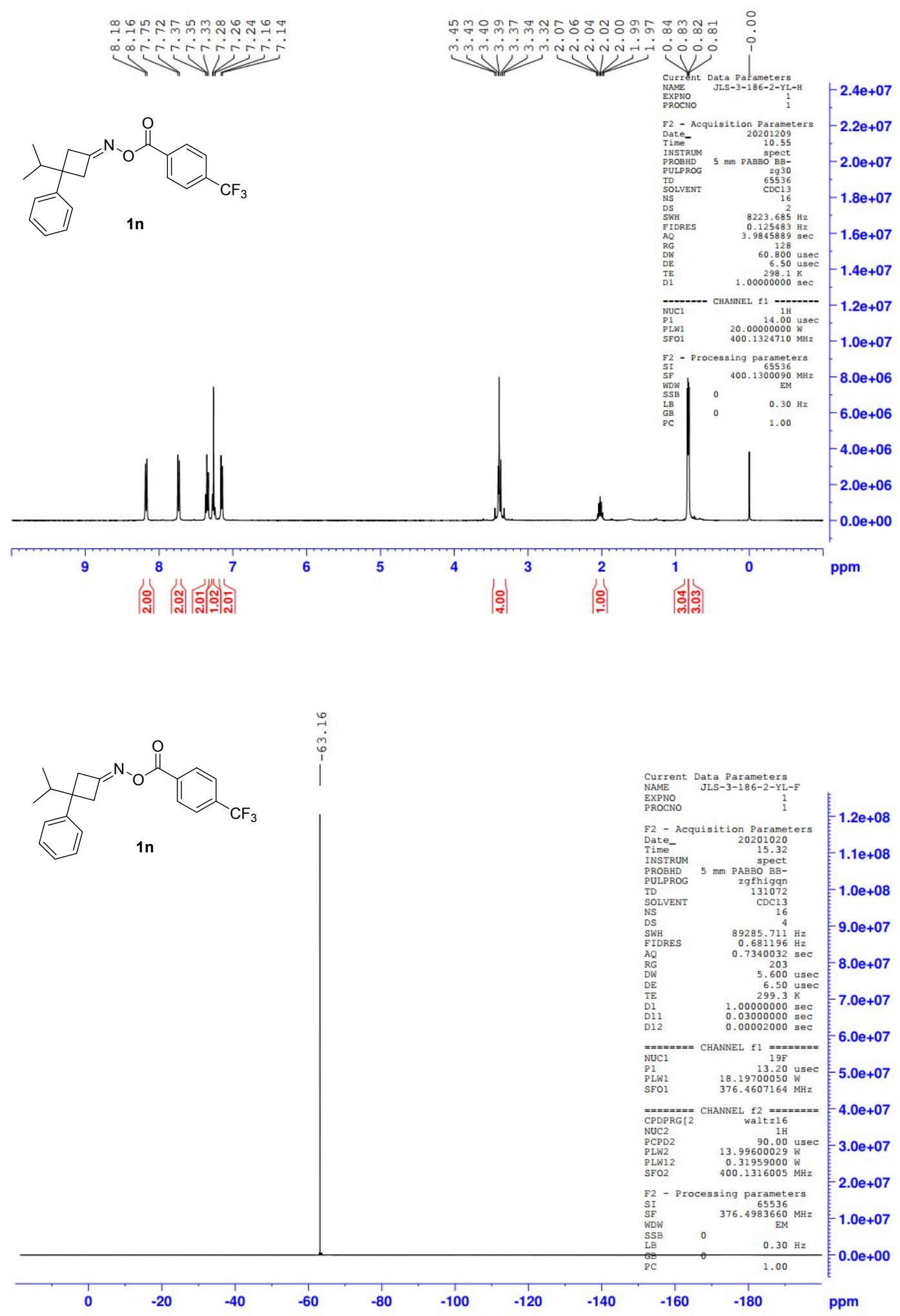

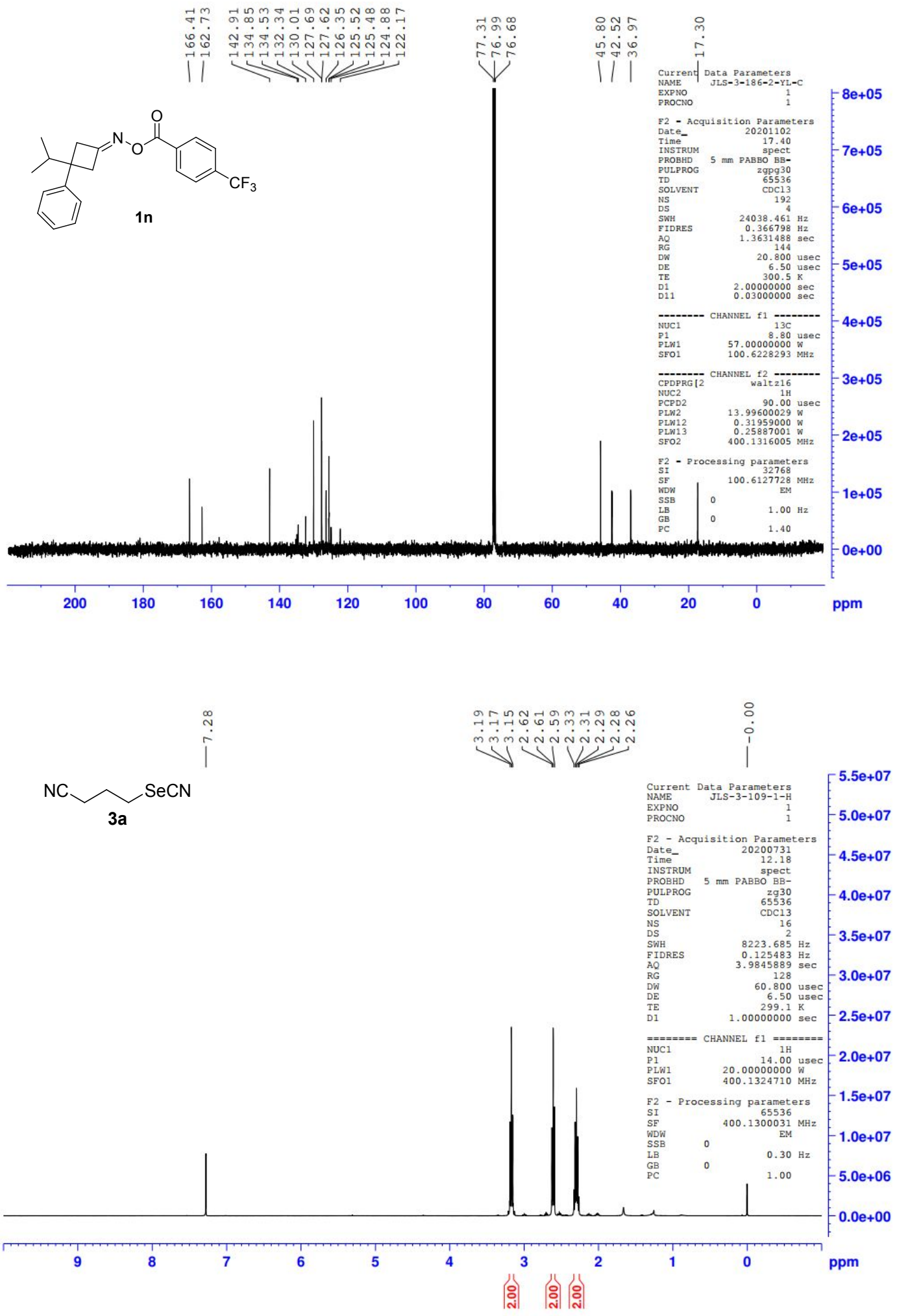

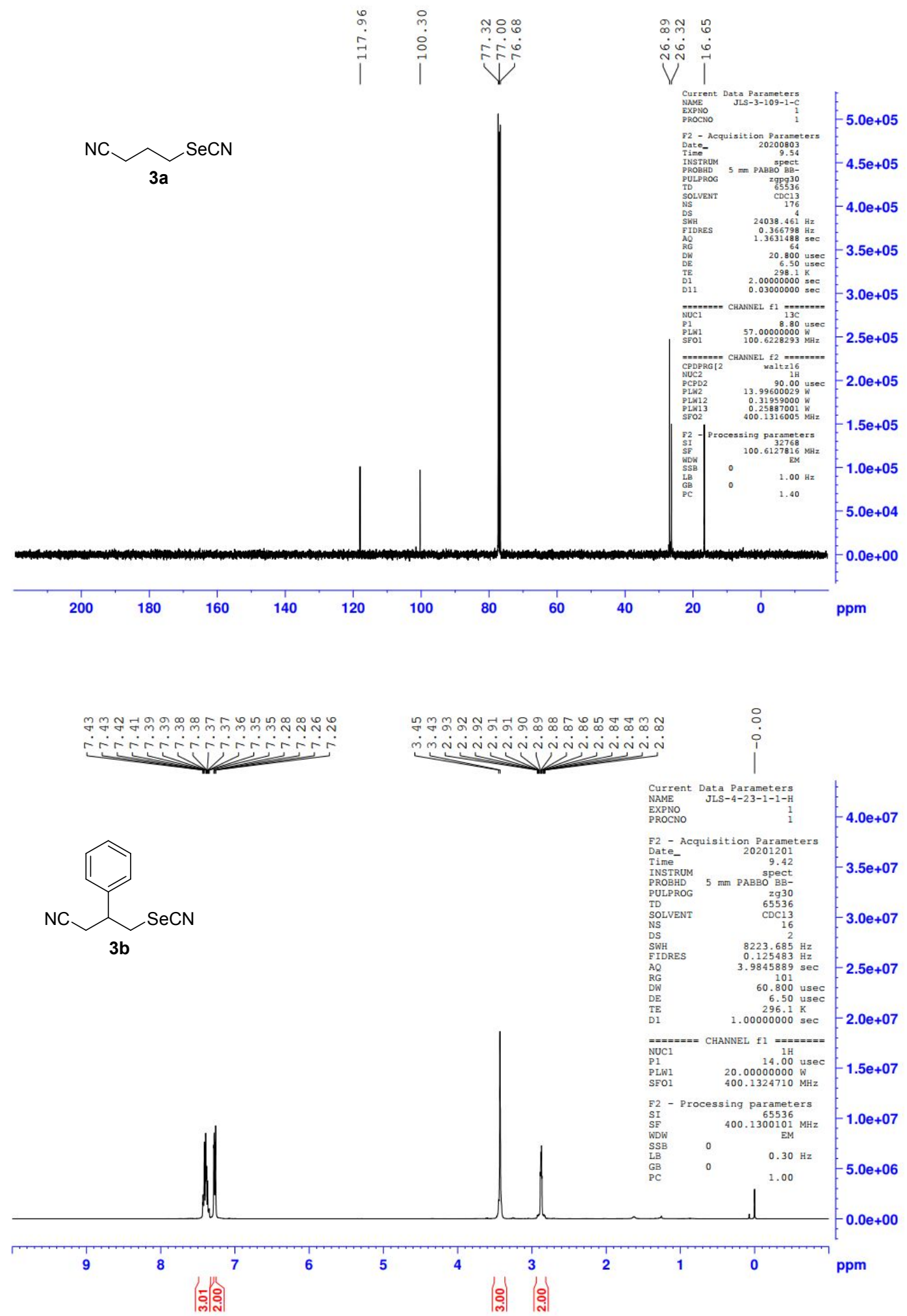

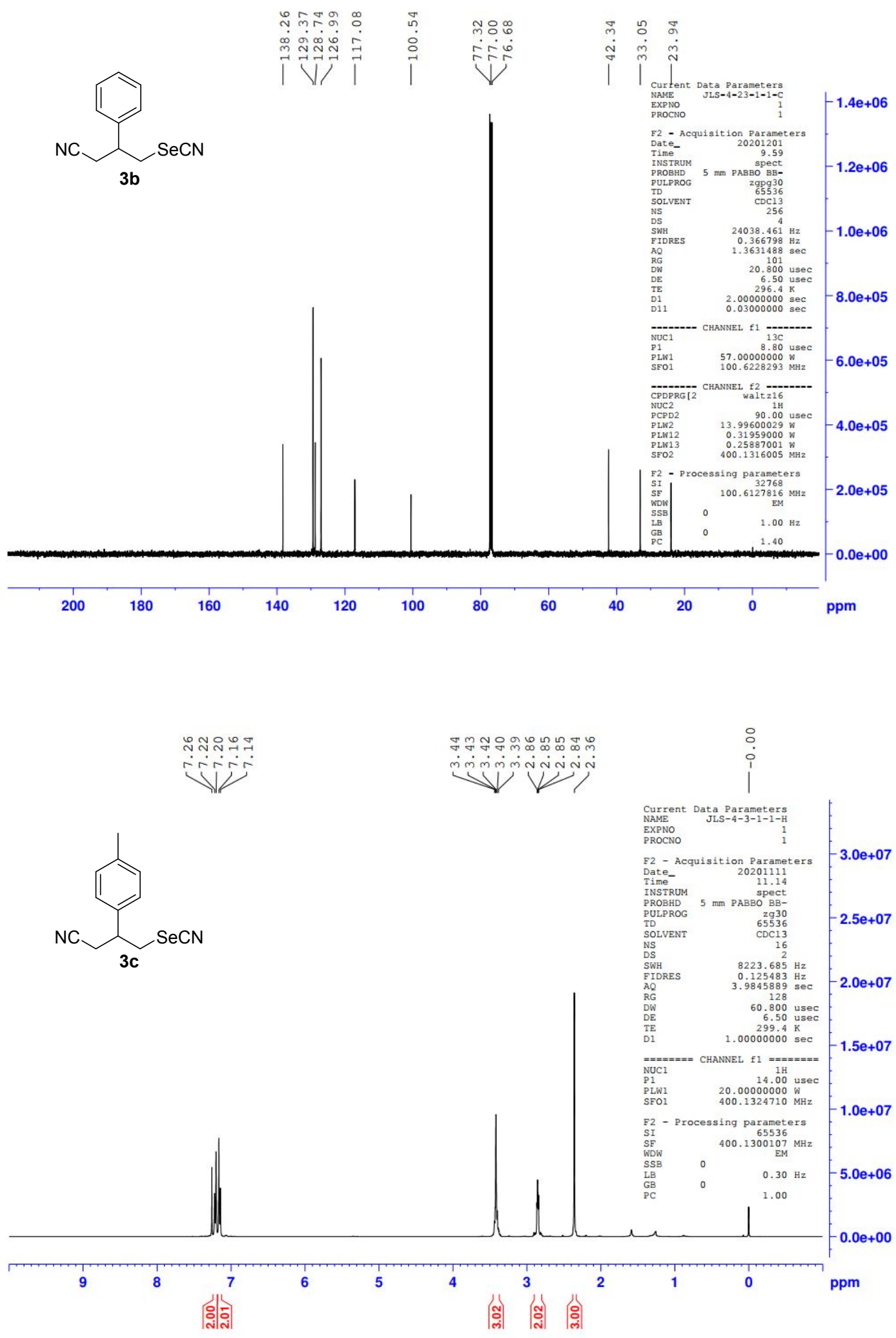

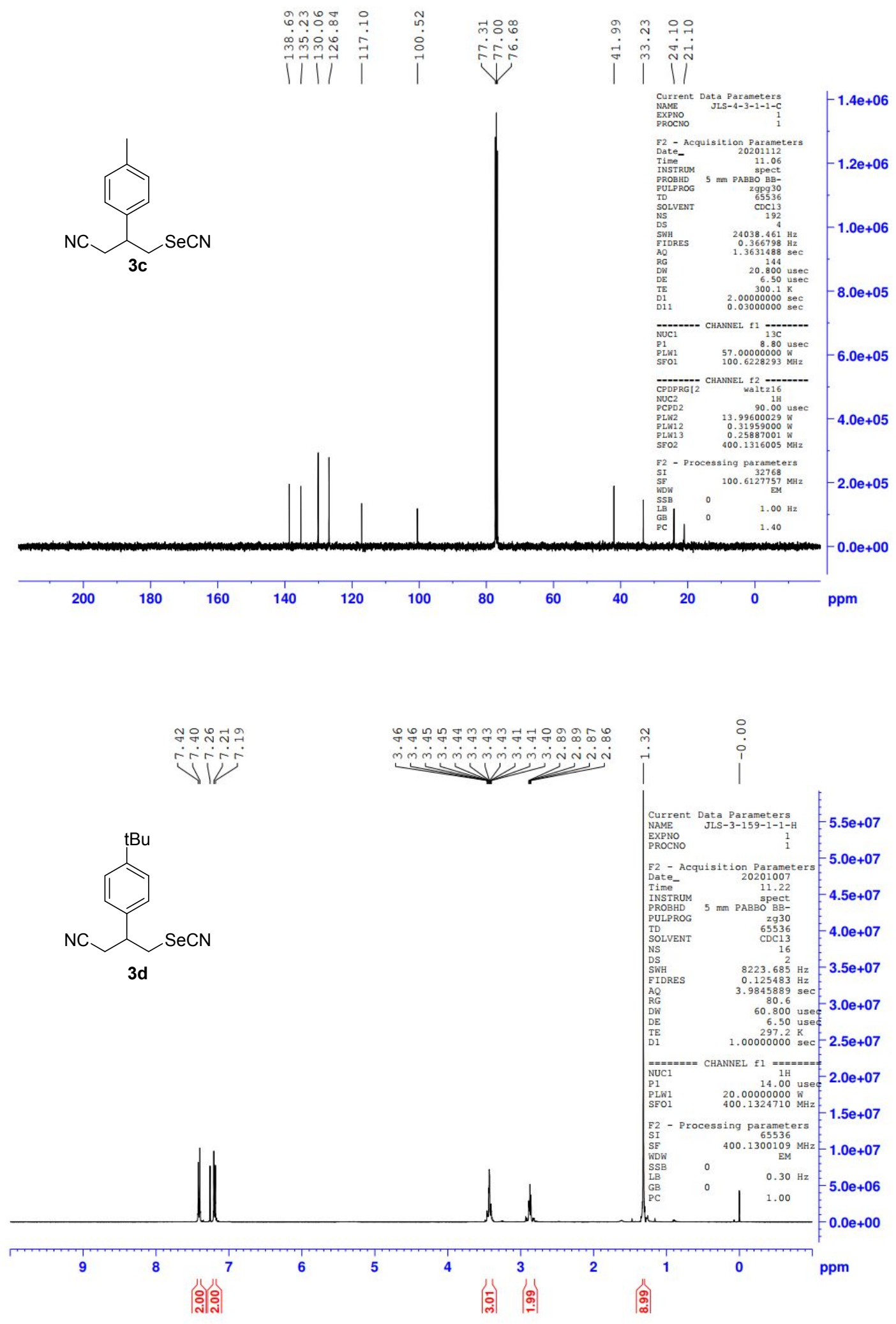

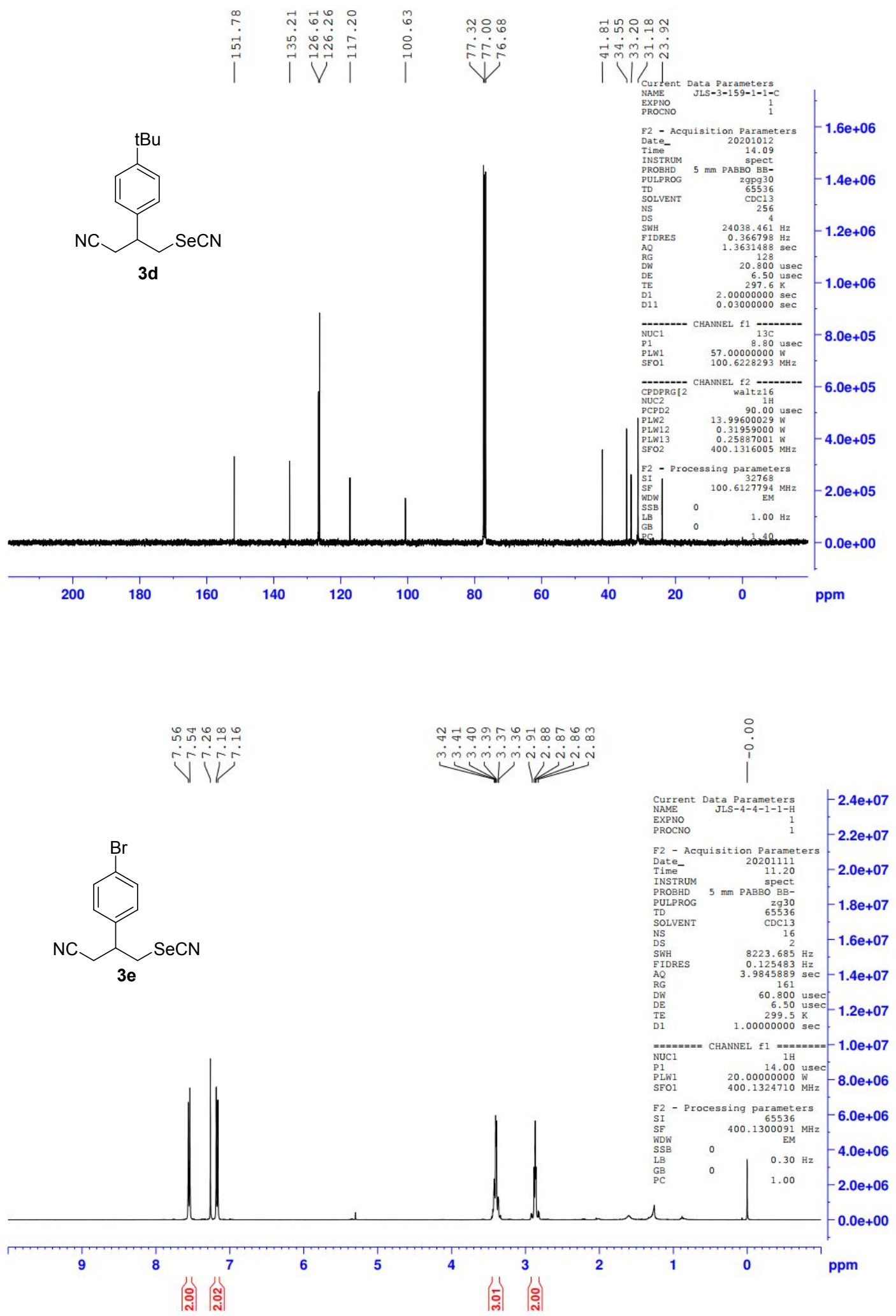

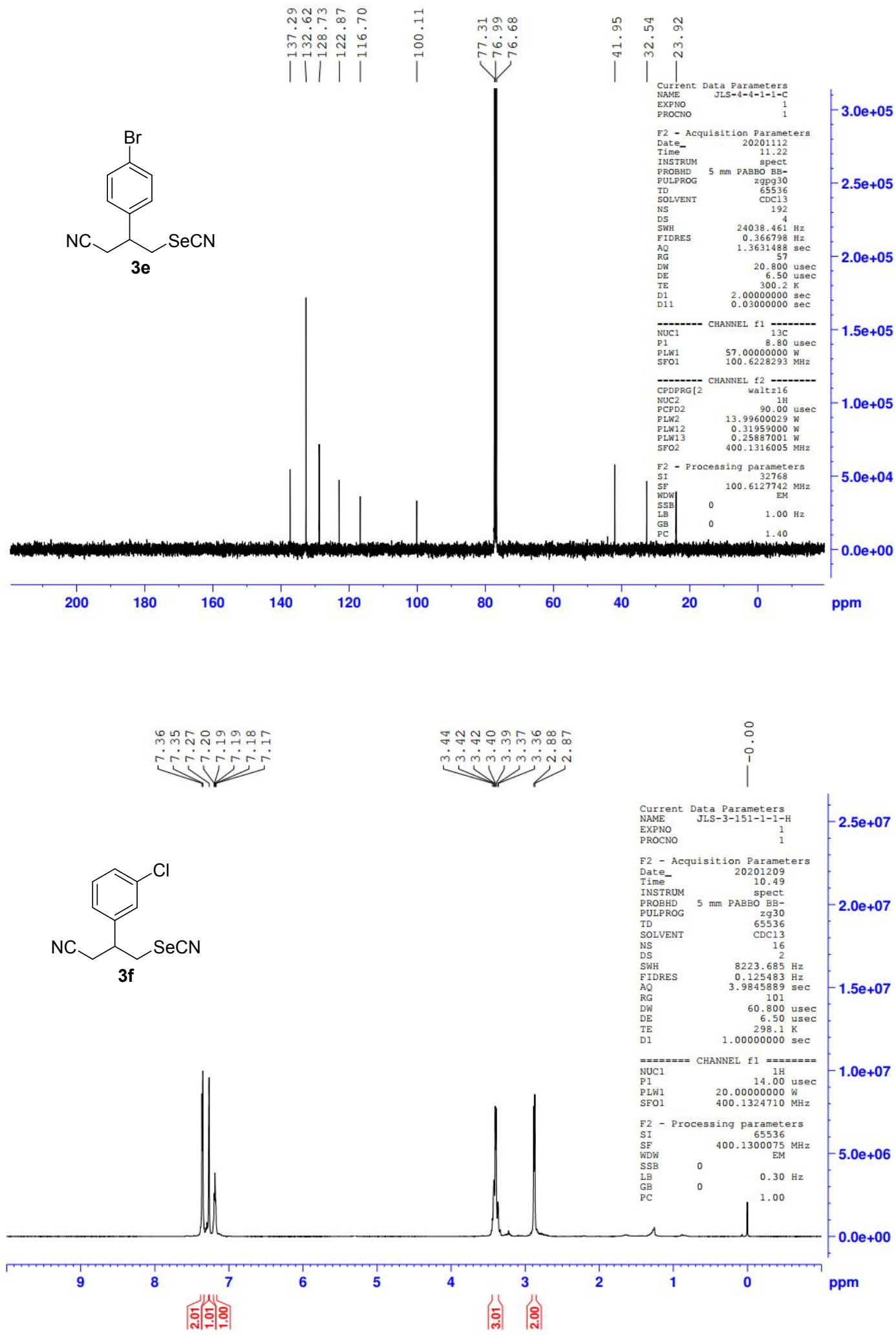

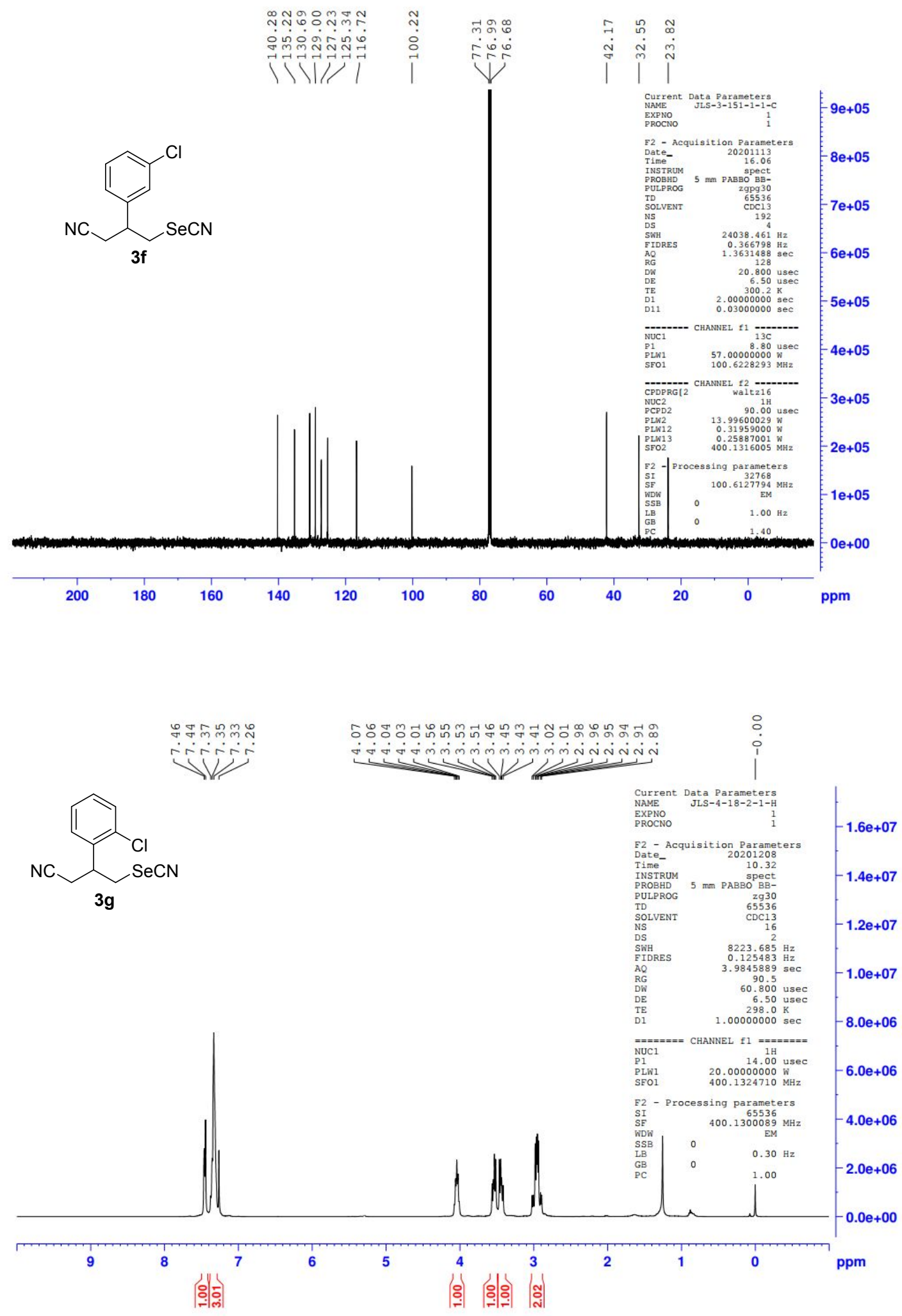

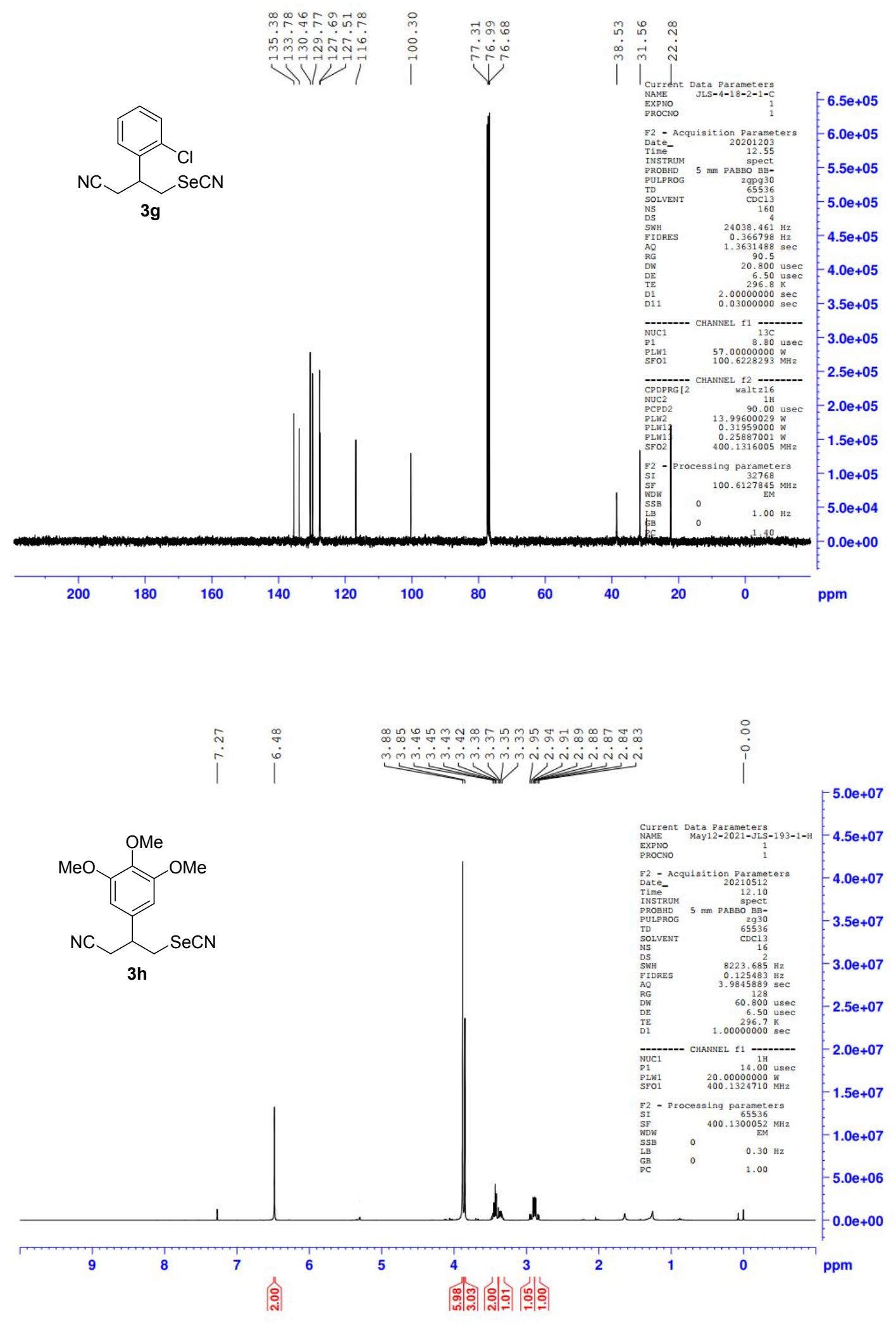


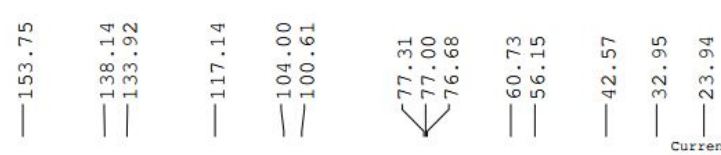
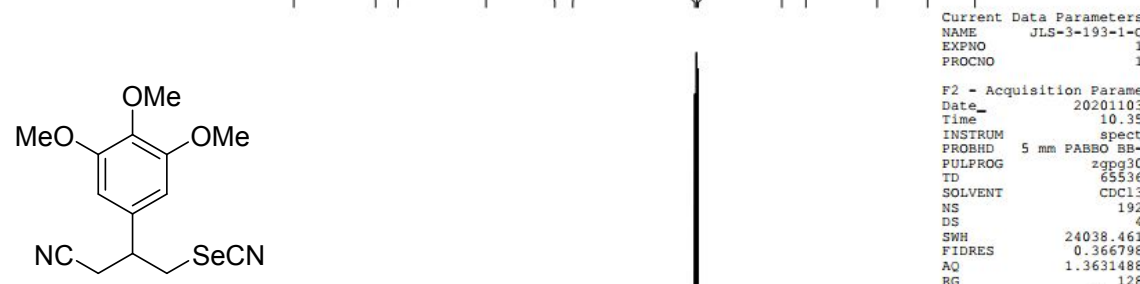

$1.2 e+06$

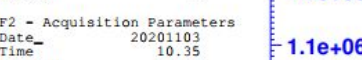

INSTRUM
PROBHD

\begin{tabular}{lr|} 
PULPROG & $29 \mathrm{gg} 30$ \\
TD & 5536 \\
SOLVENT & $\mathrm{CDC13}$
\end{tabular}

\begin{tabular}{lc|l} 
NS \\
DS \\
SWH & 192 & 192 \\
SW & $24038.461 \mathrm{~Hz}$ & $\mathbf{9 . 0 e + 0 5}$
\end{tabular}

$3 \mathrm{~h}$

$0.366798 \mathrm{~Hz}$
$1.3631488 \mathrm{seC}$

128 . $8.00+05$ $8.0 \mathrm{e}+05$

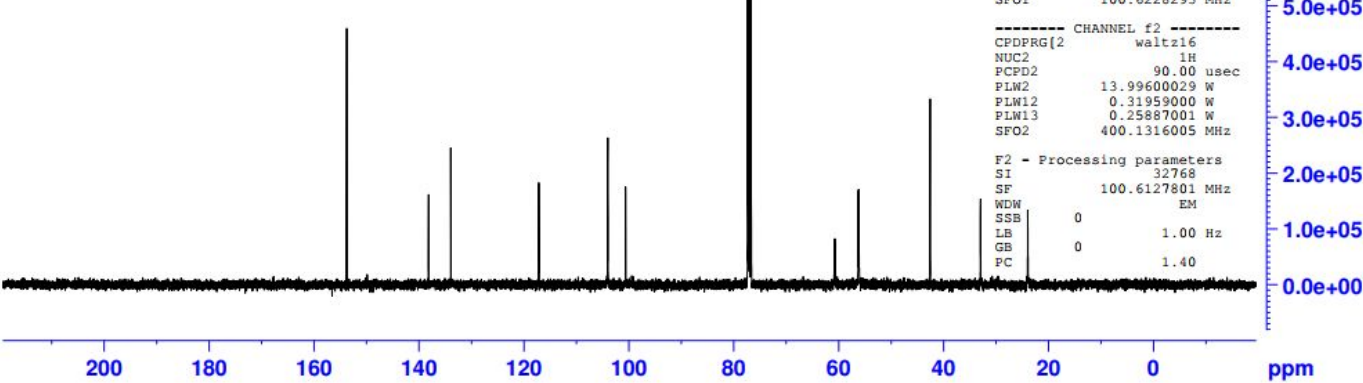

$4.0 \mathrm{e}+05$

$3.0 \mathrm{e}+05$

$.0 \mathrm{e}+05$

200

ppm

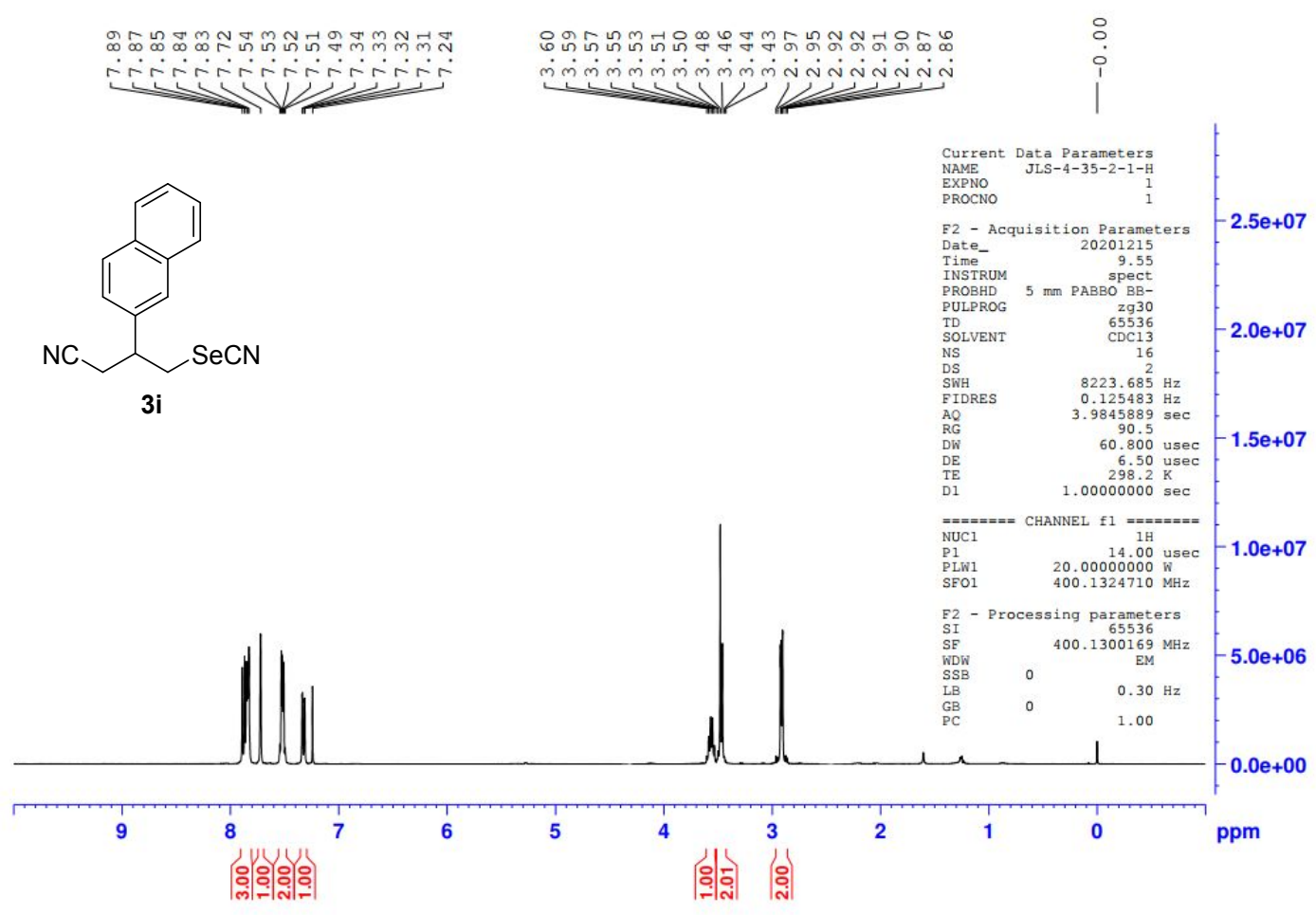



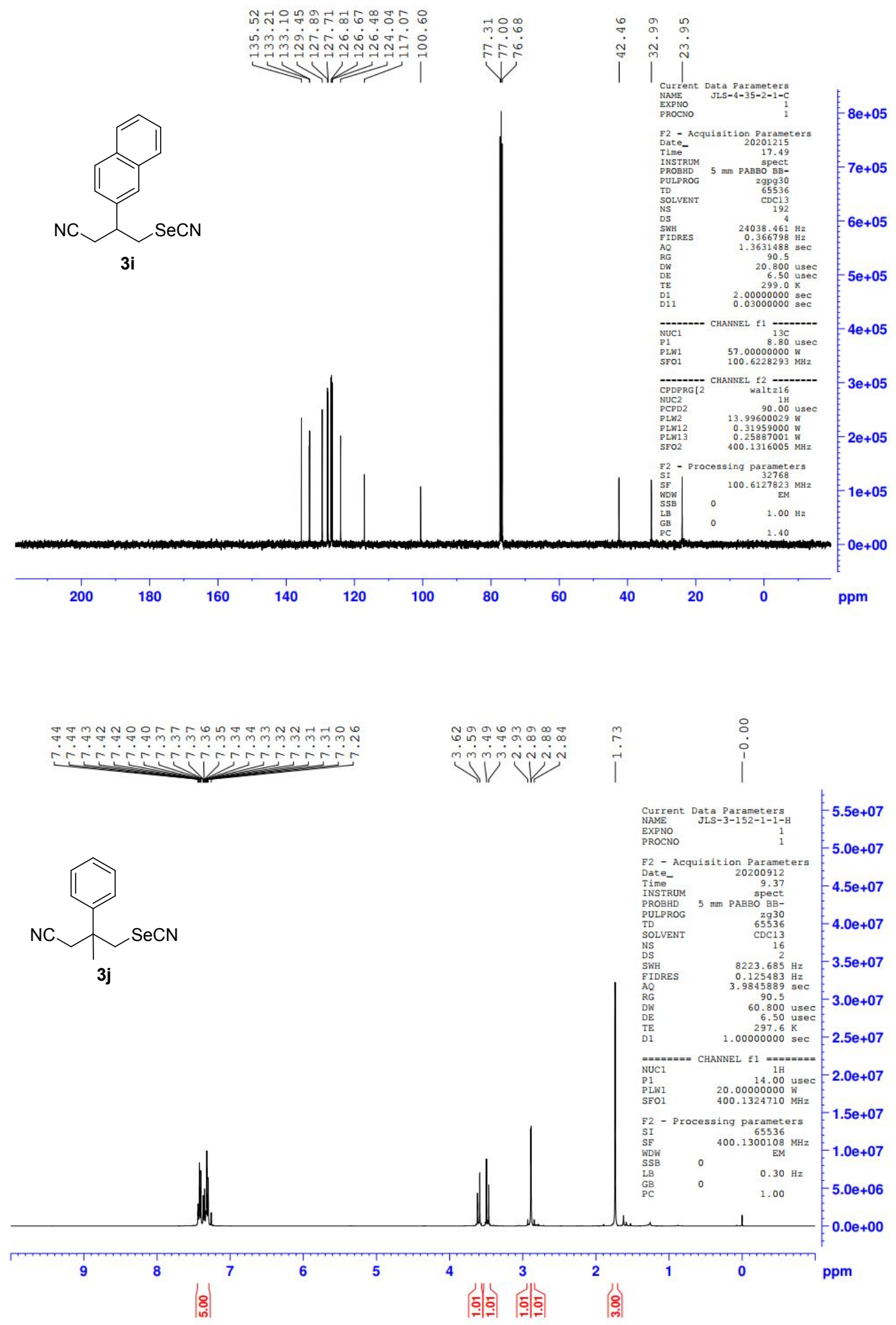

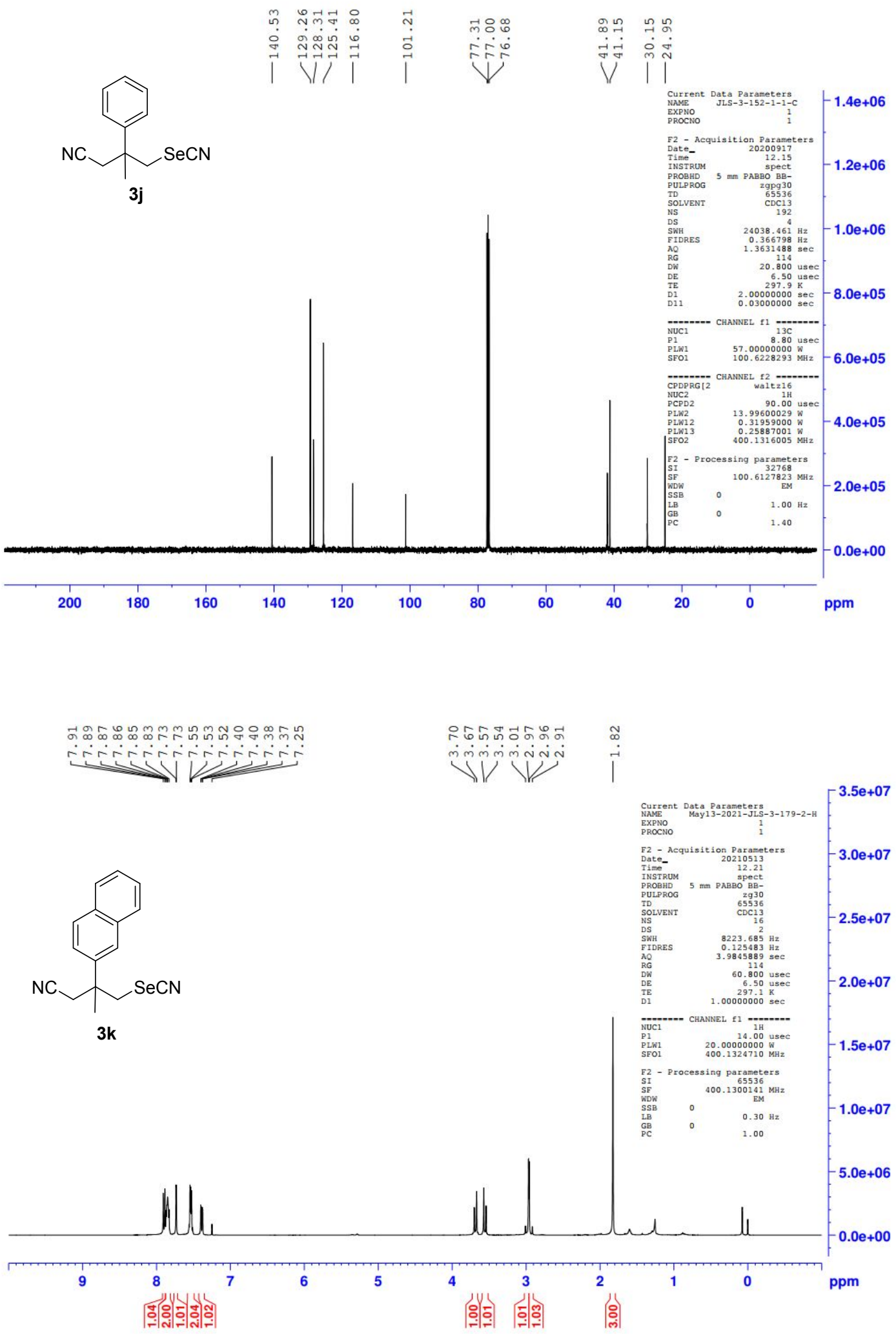

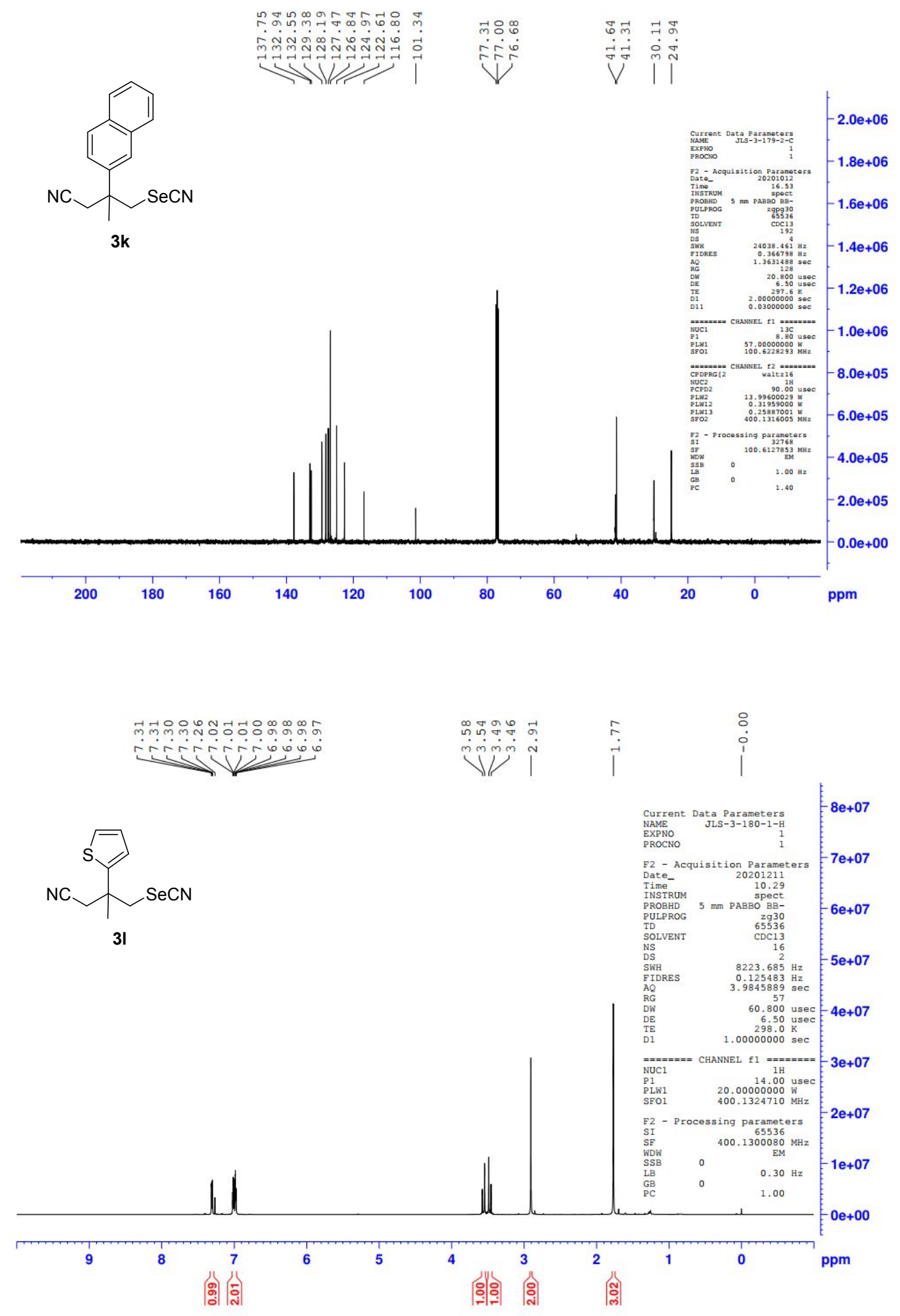

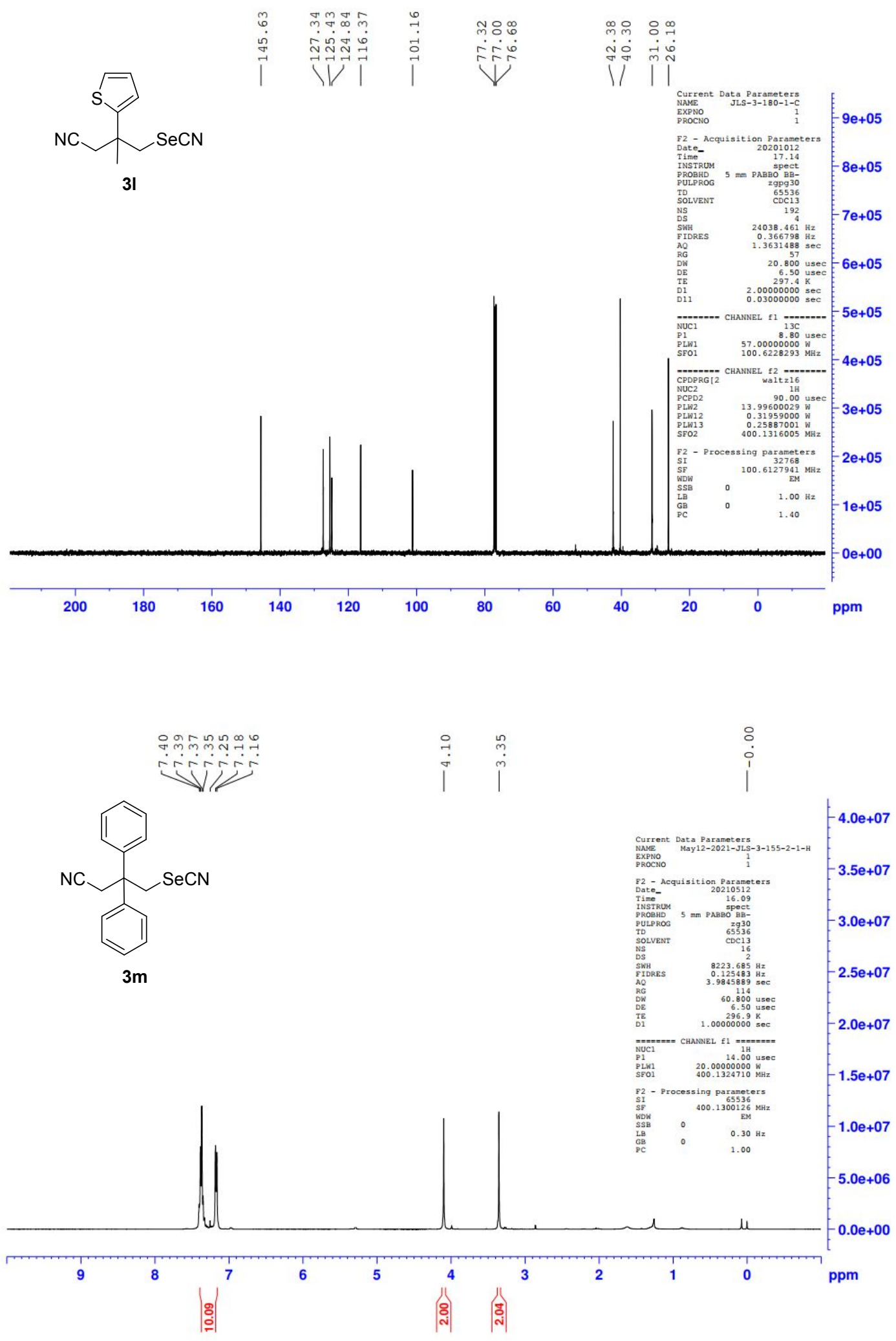

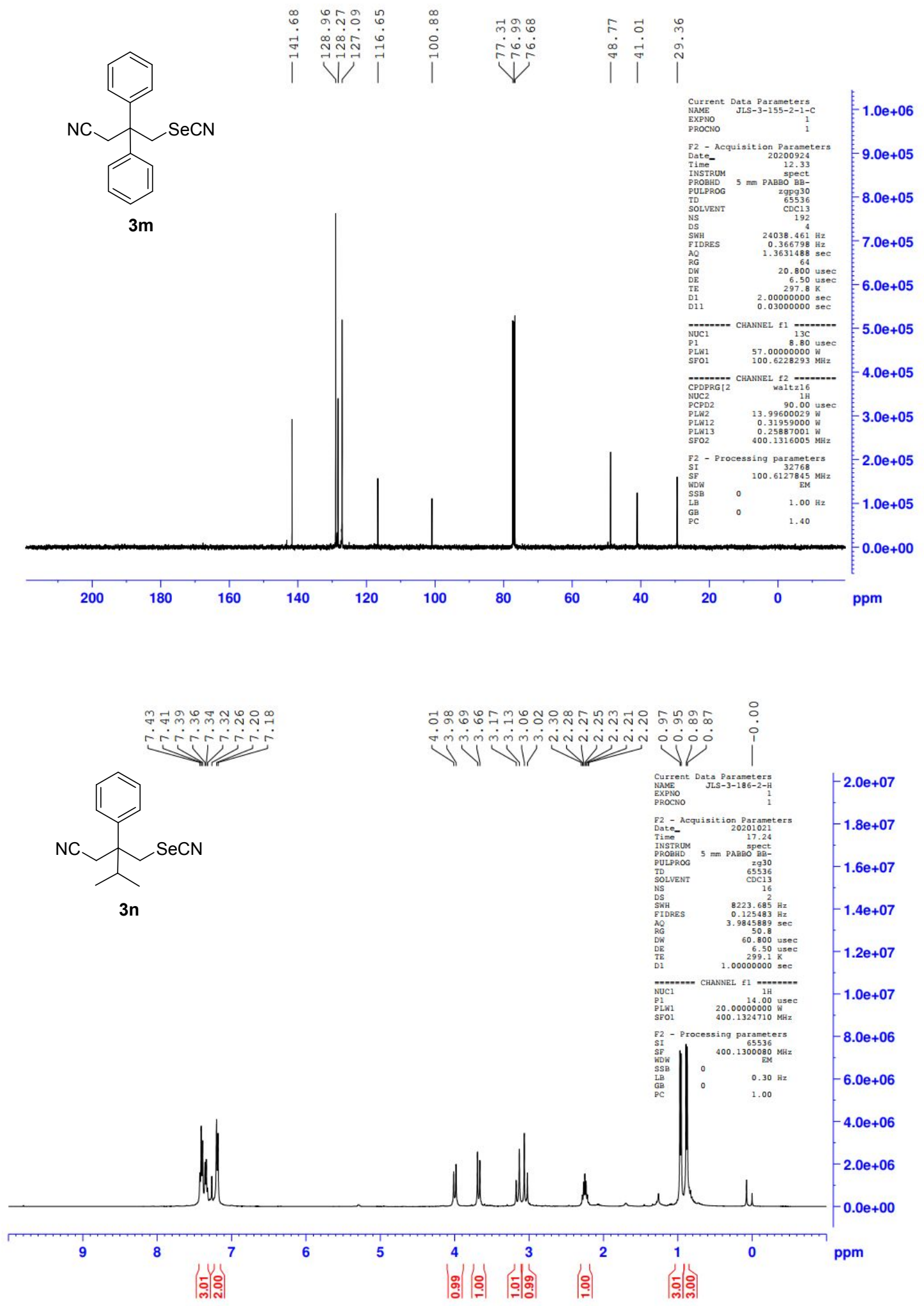

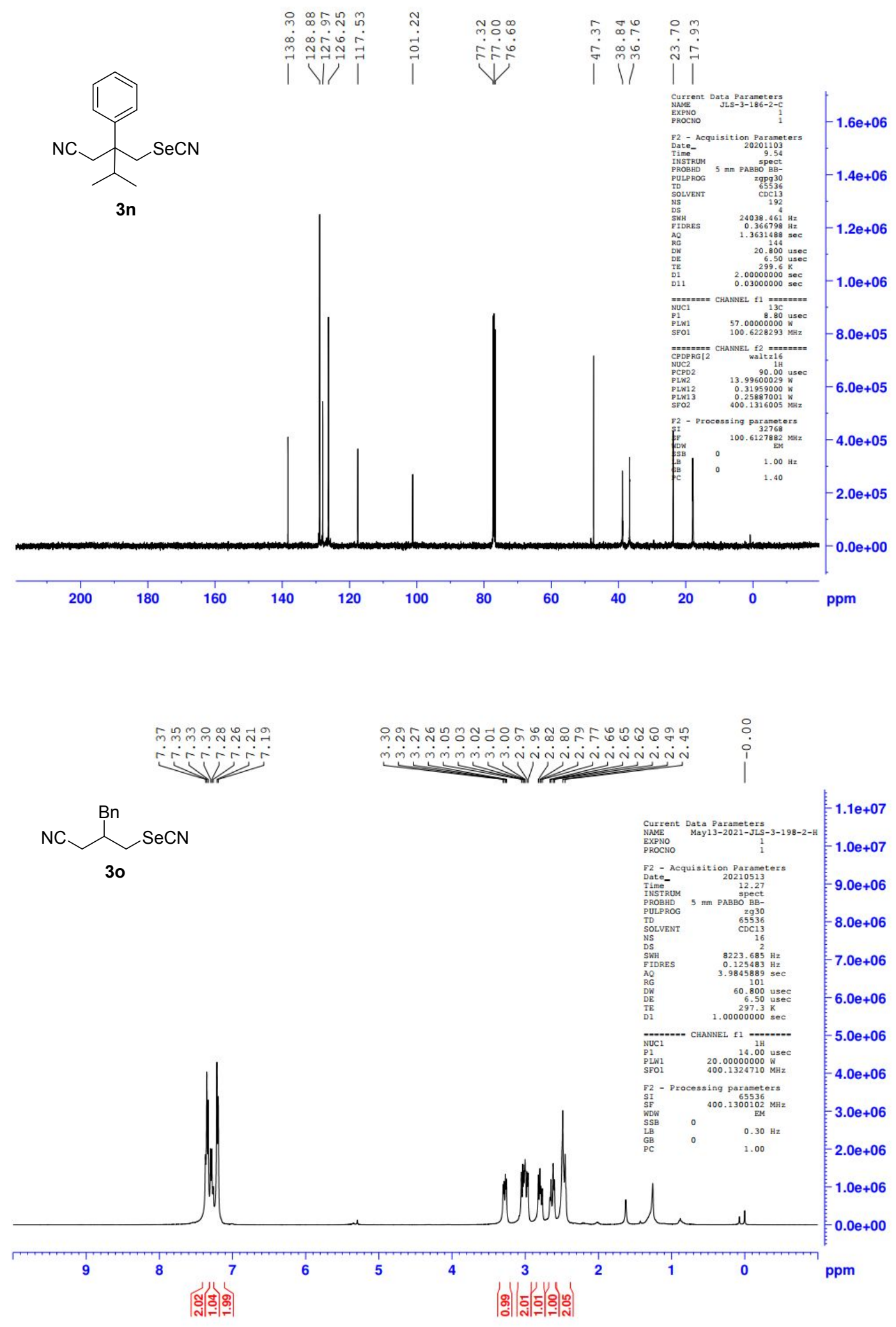

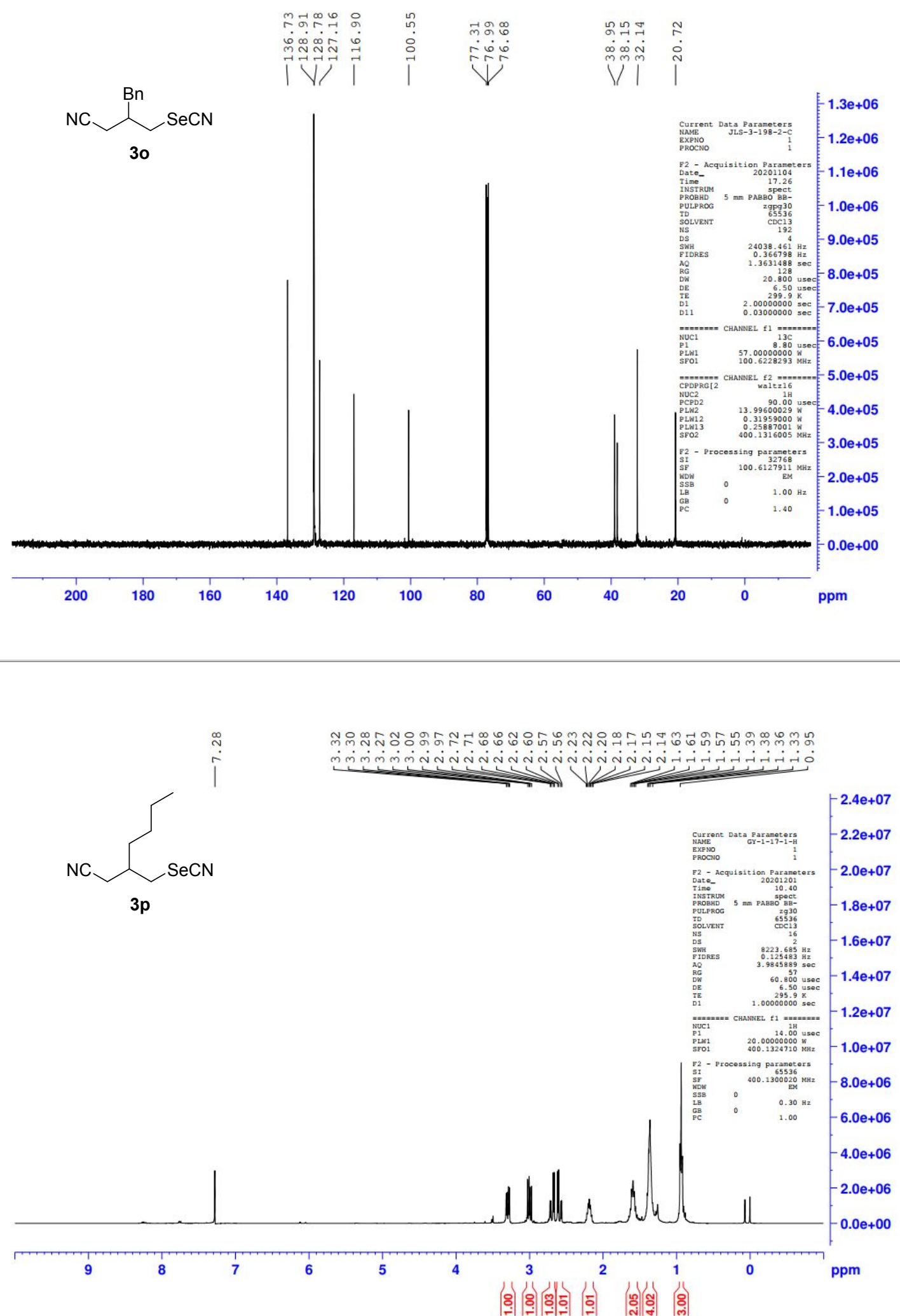

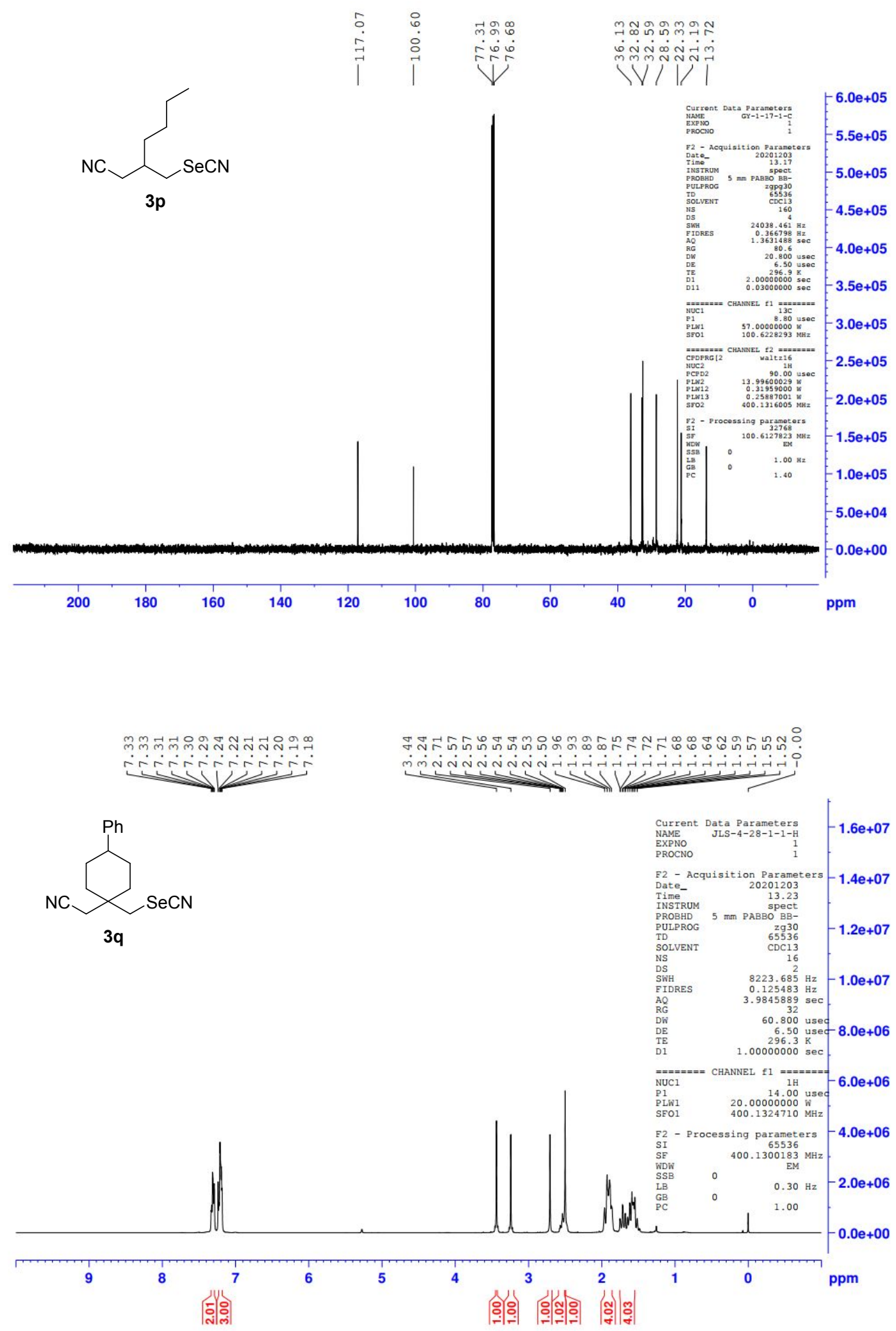

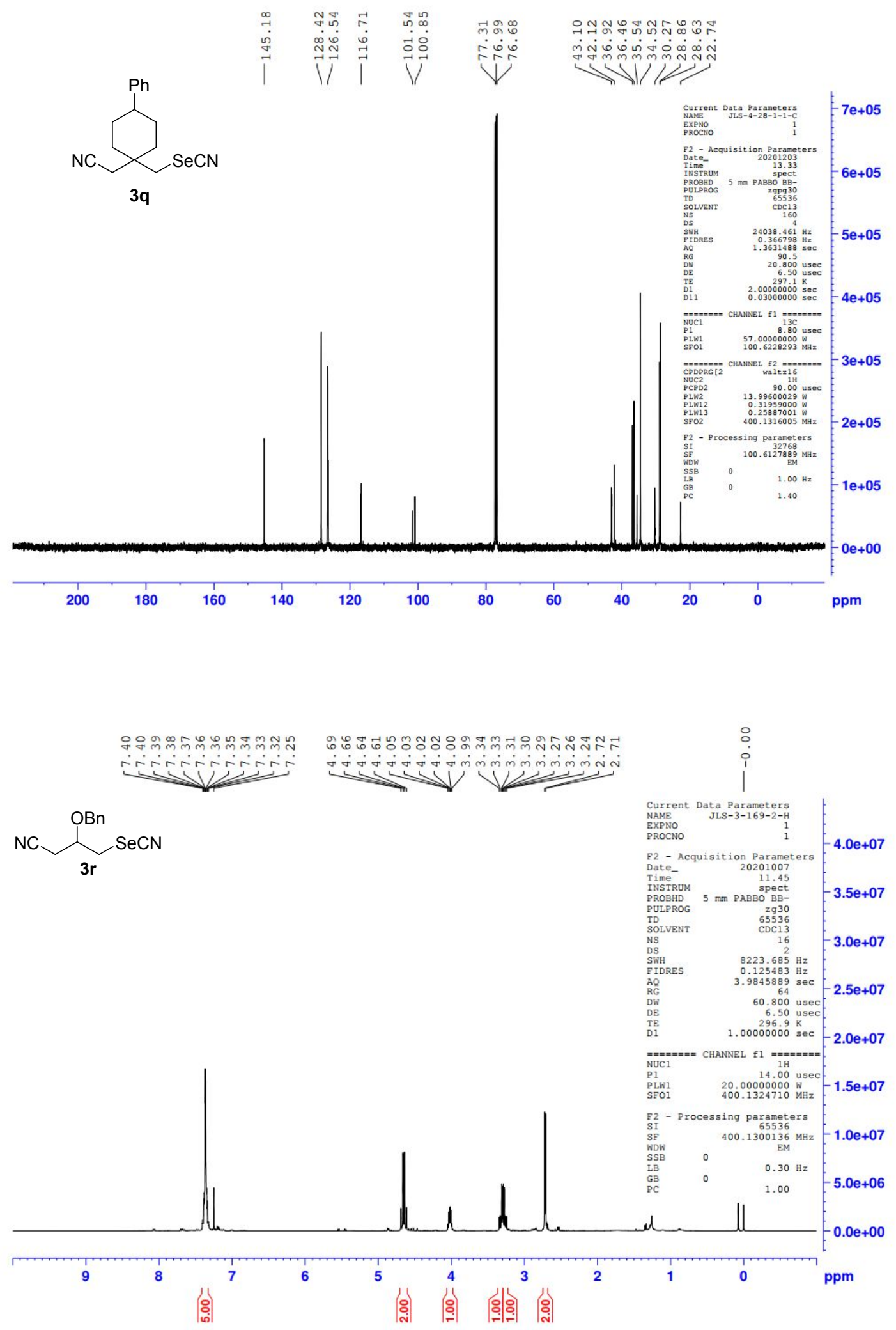

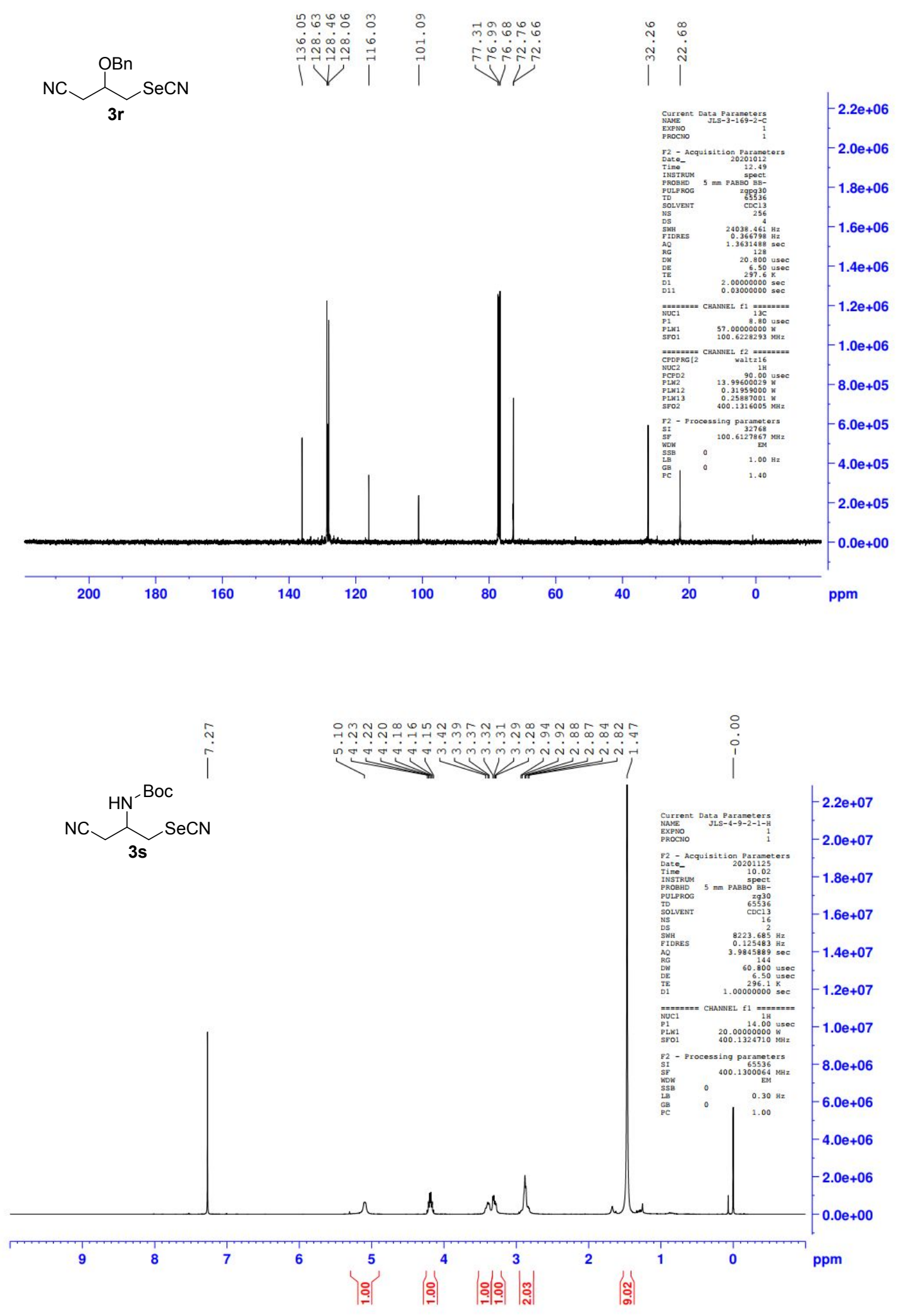

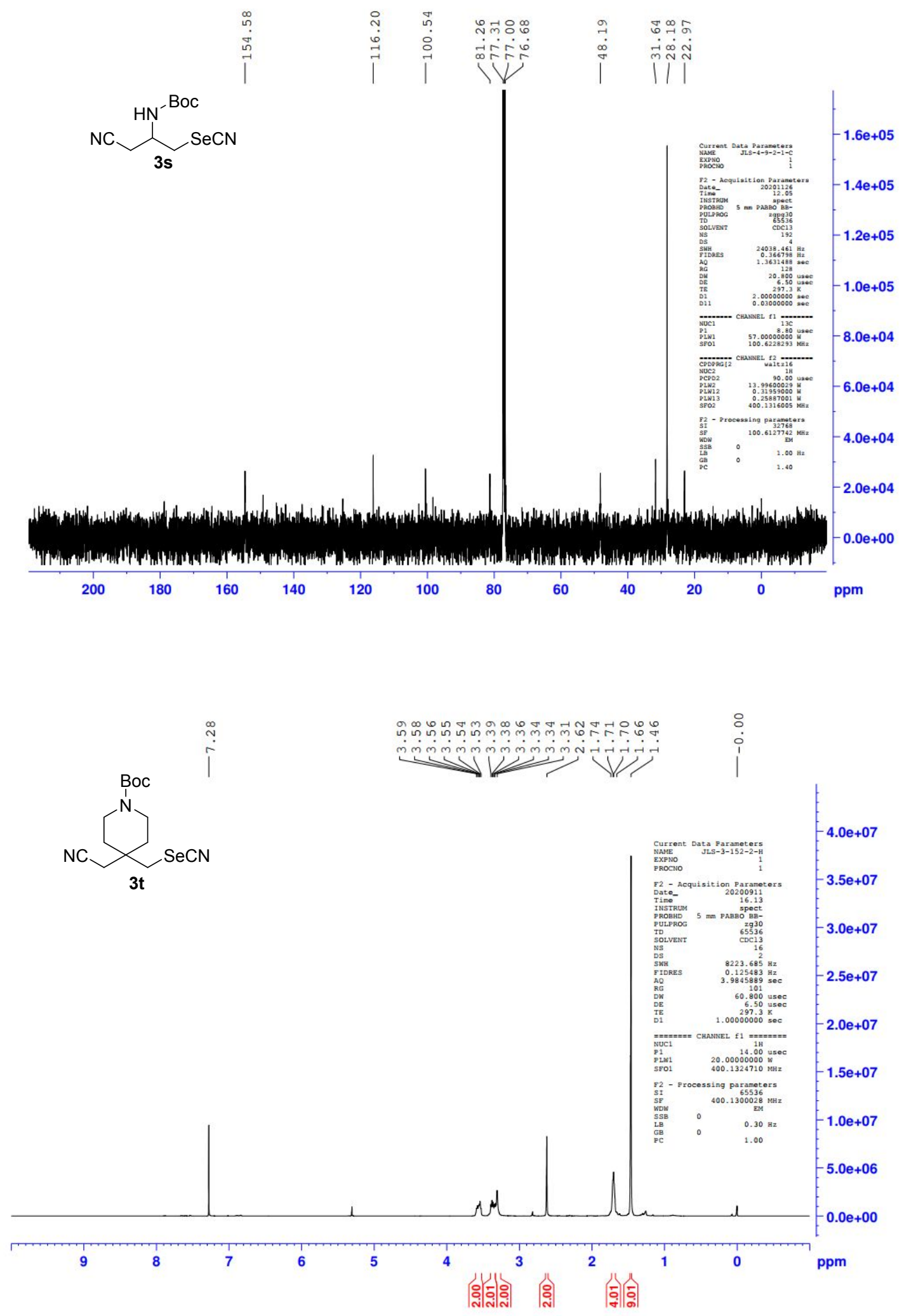

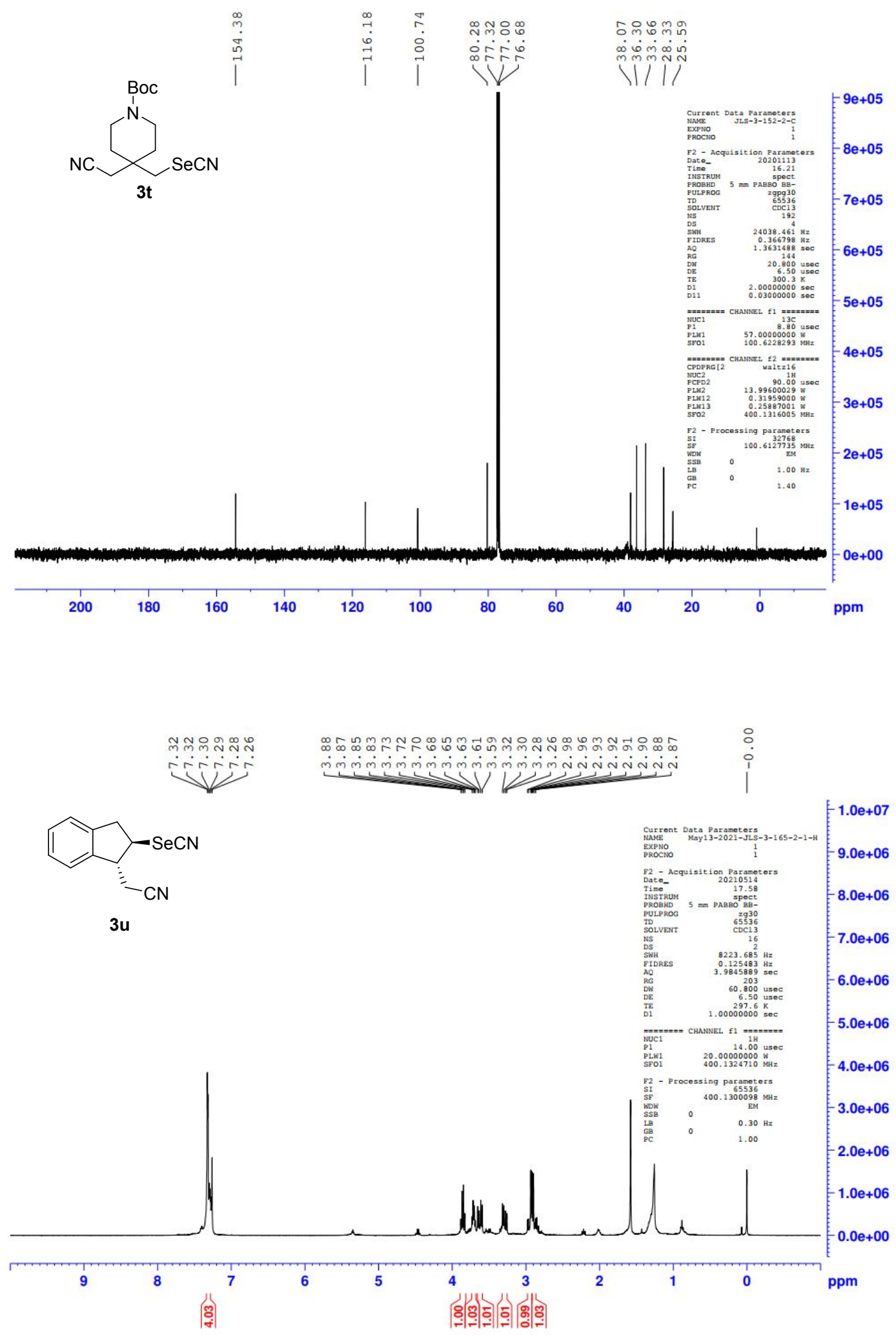

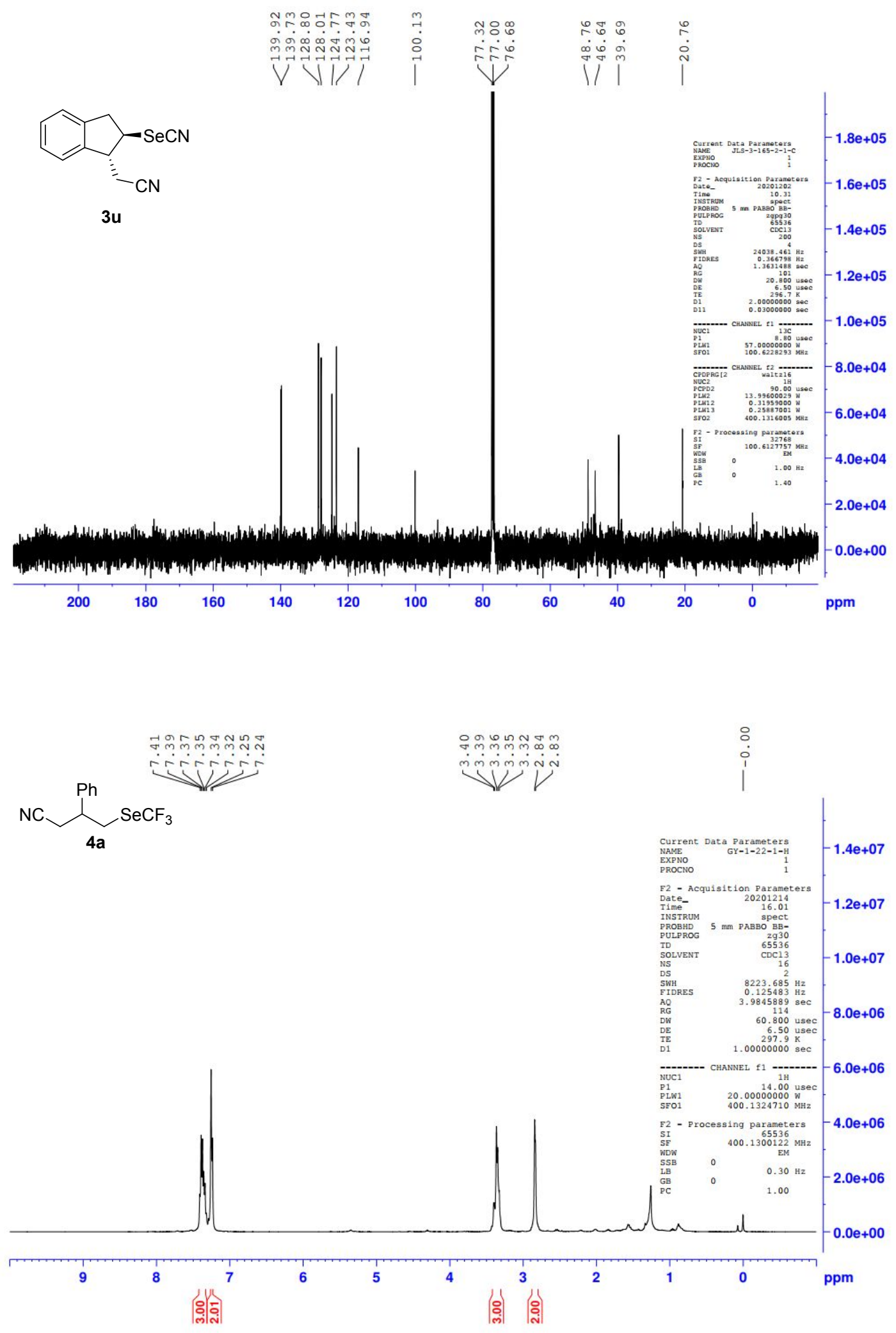

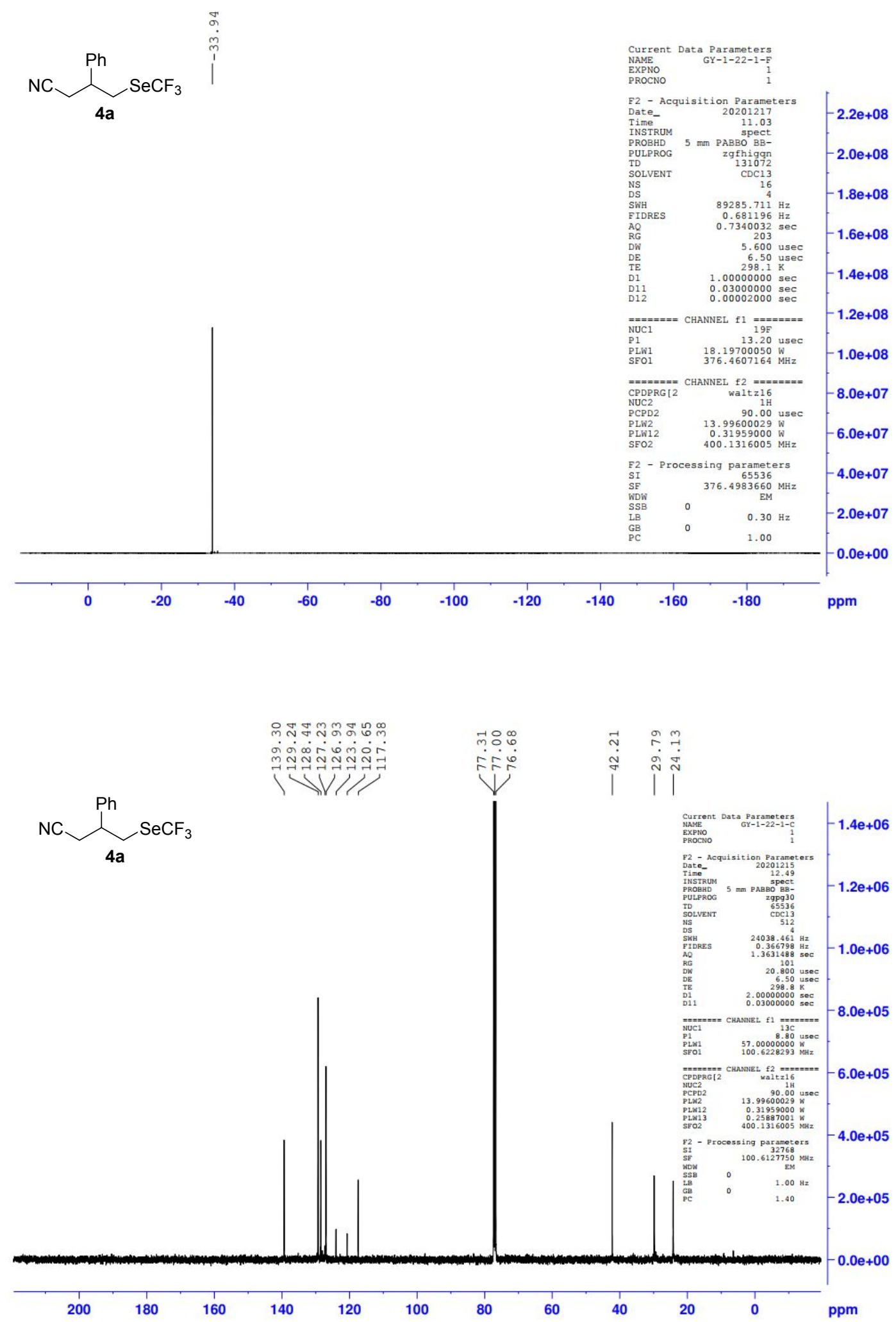

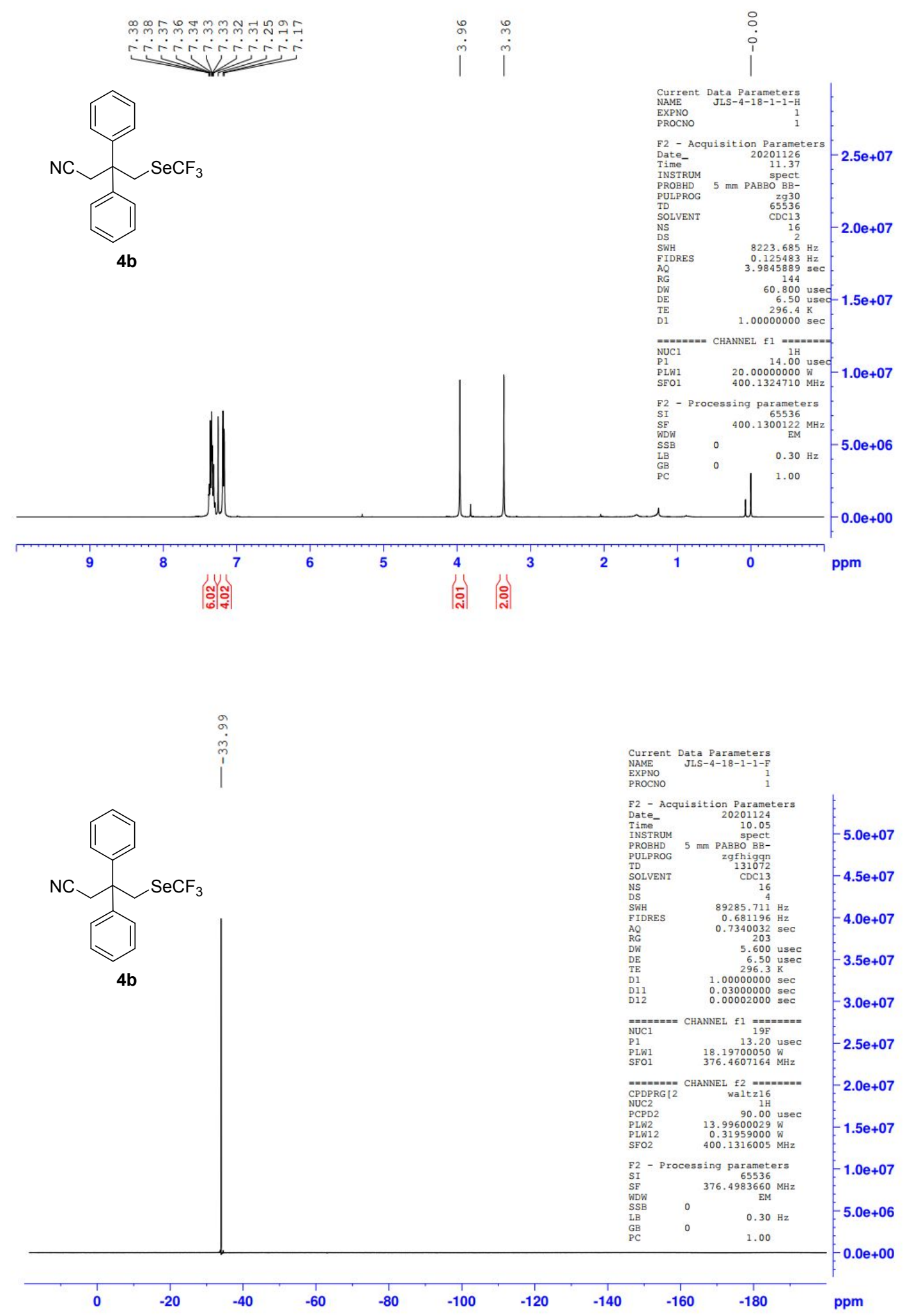

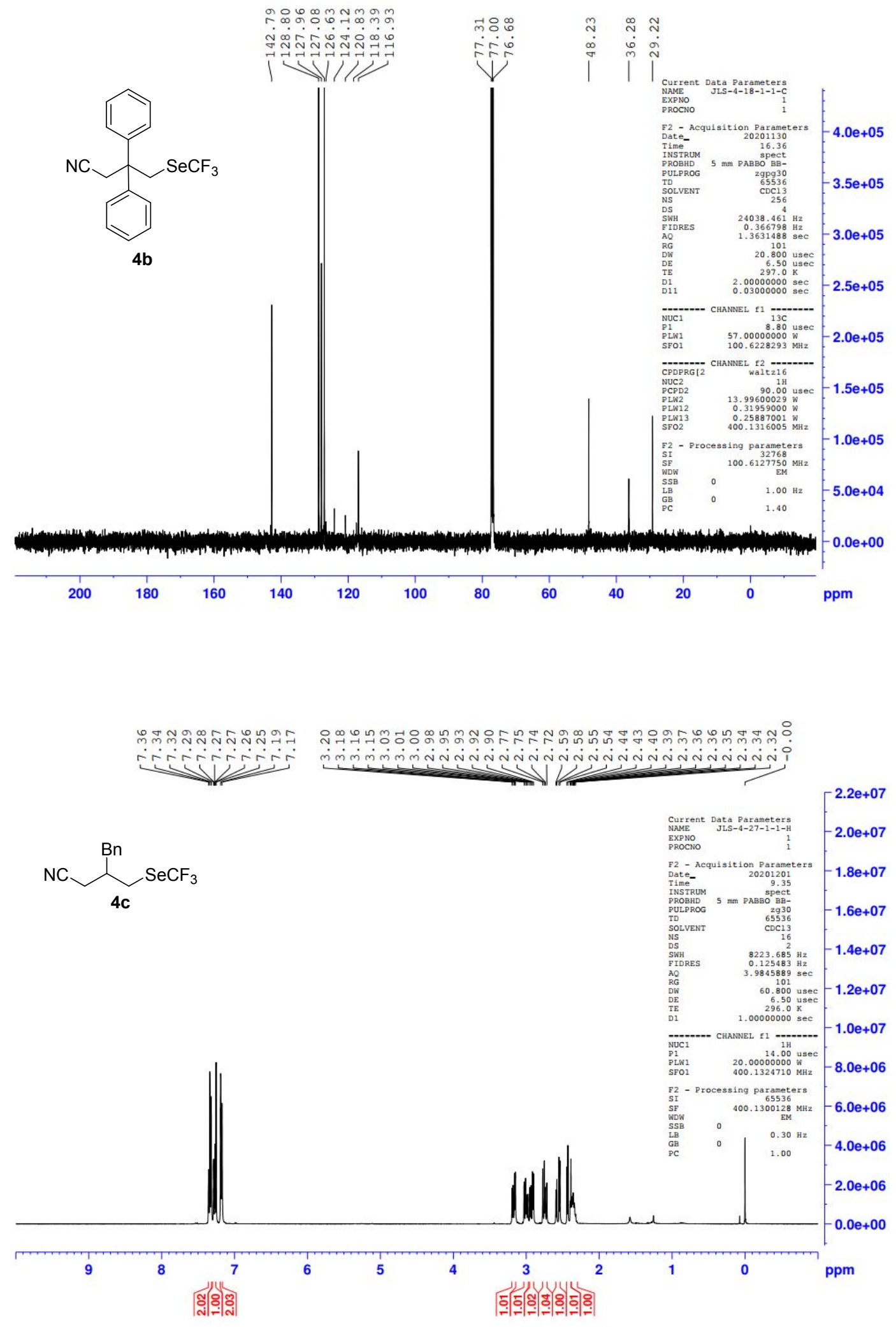

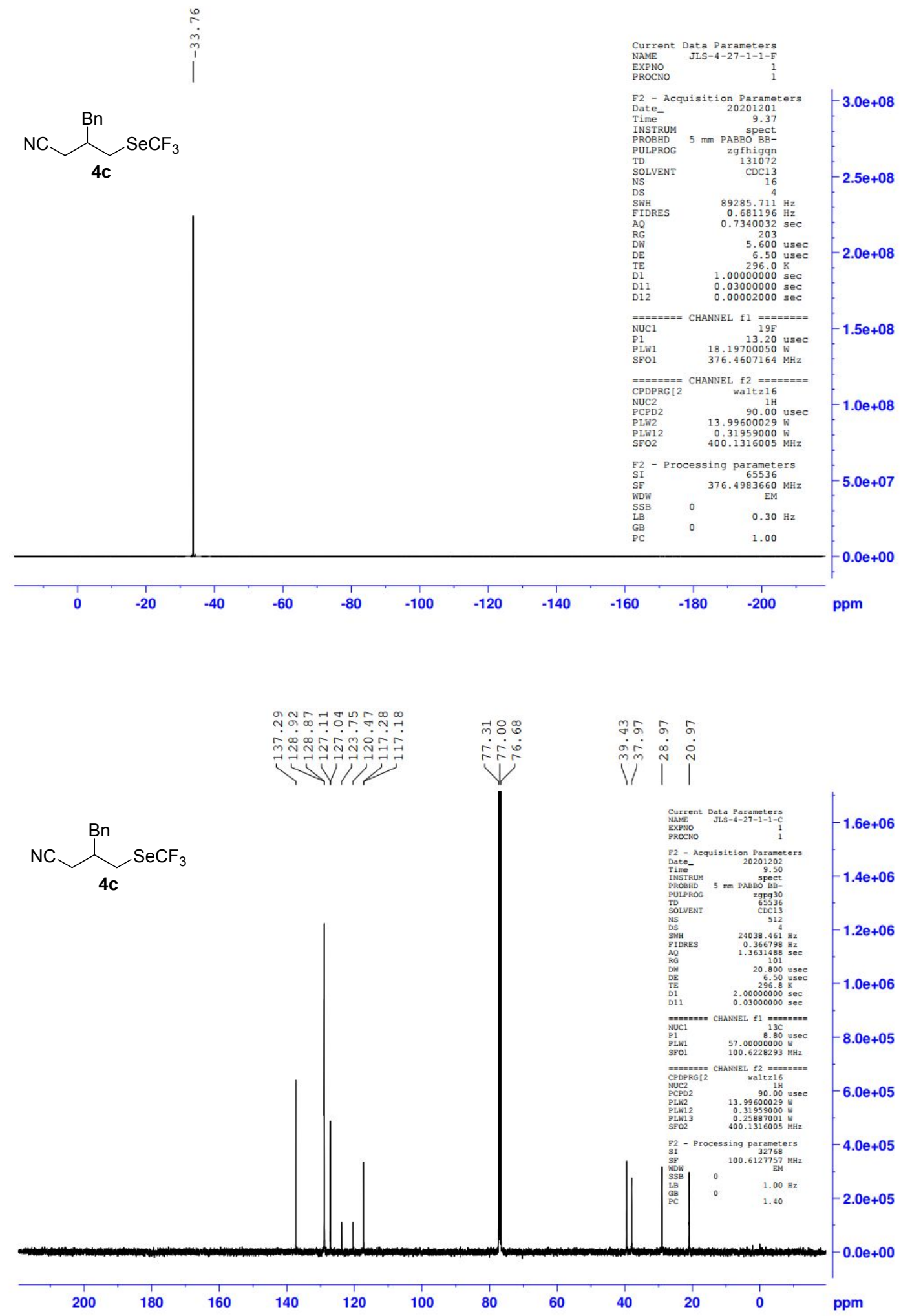

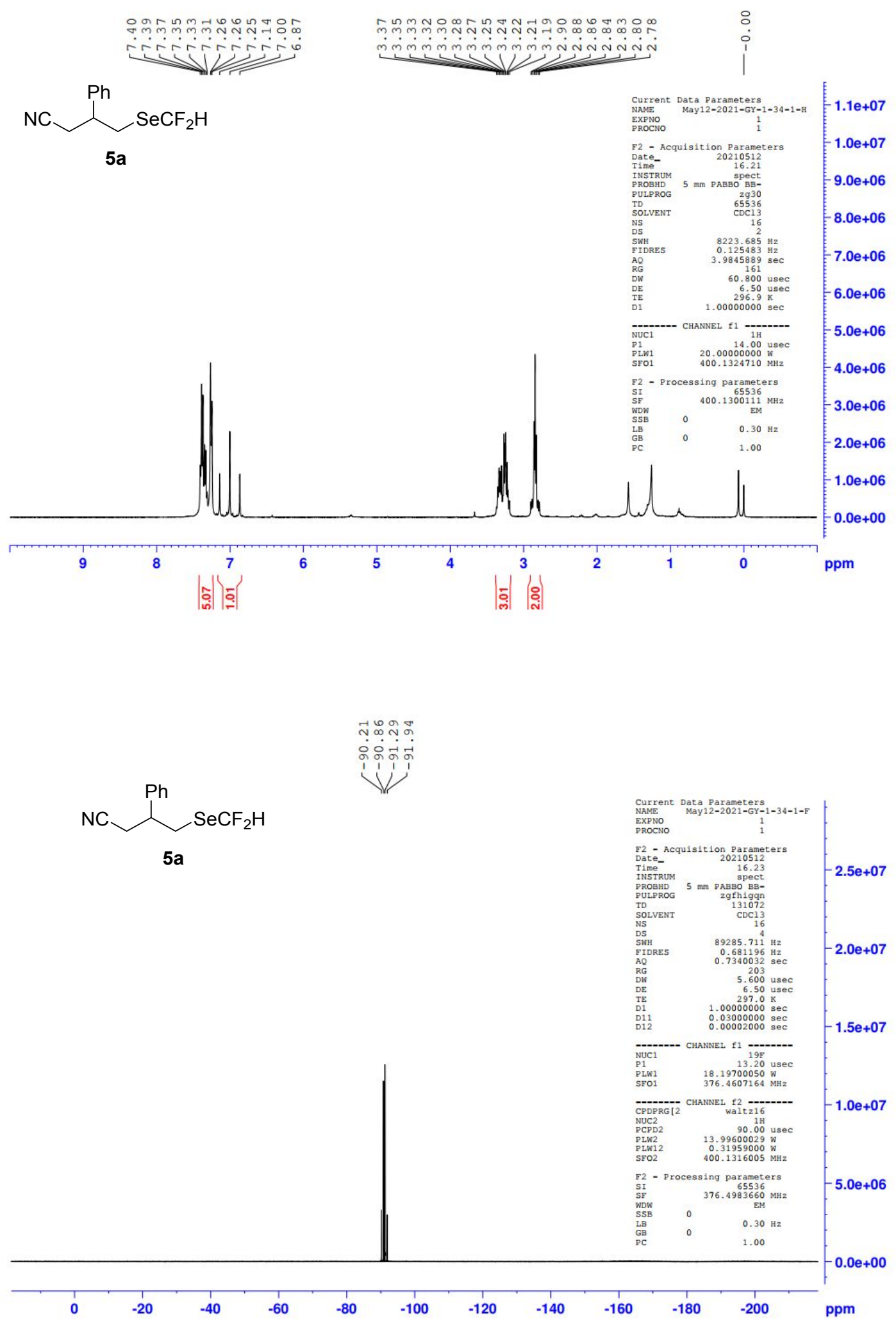

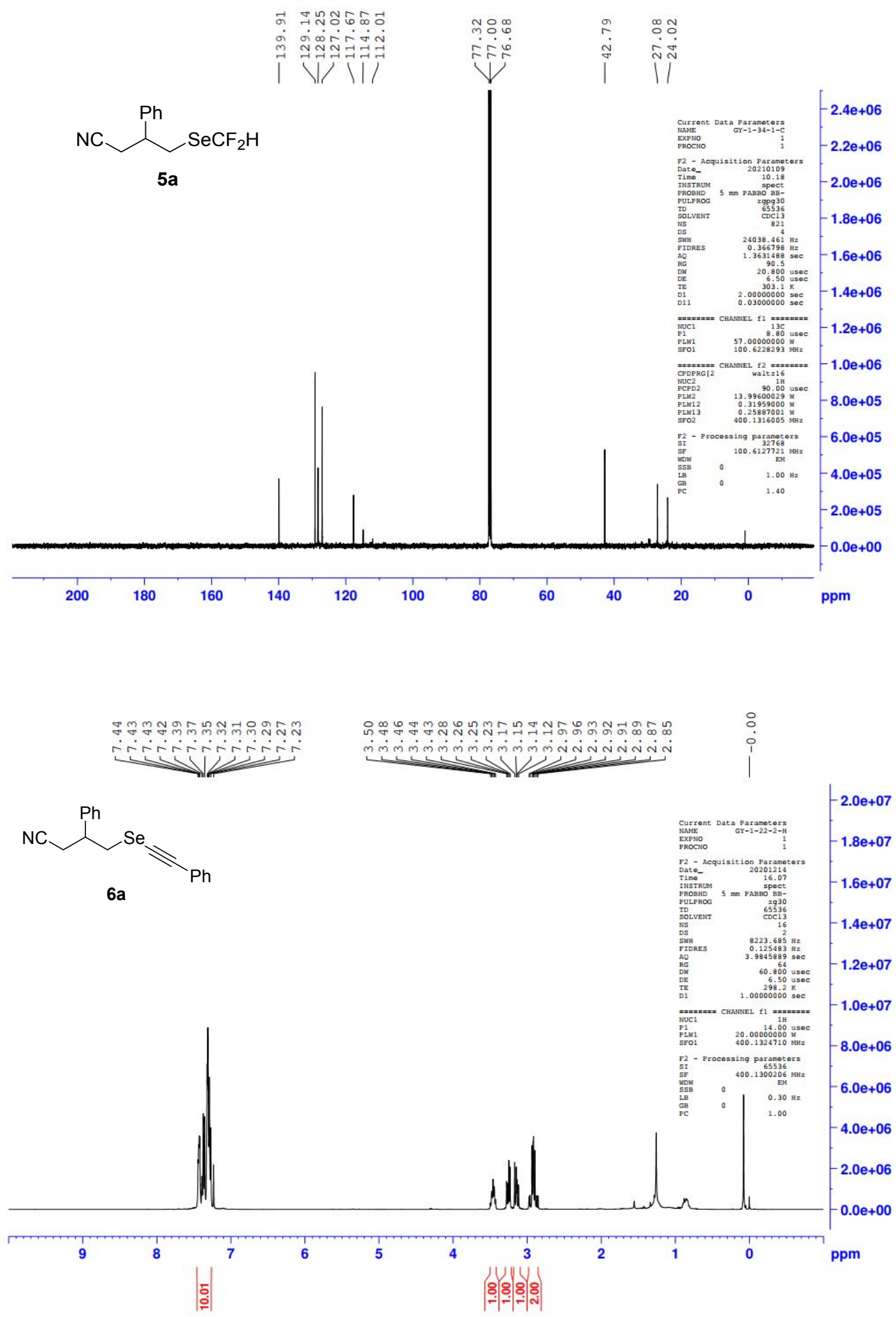

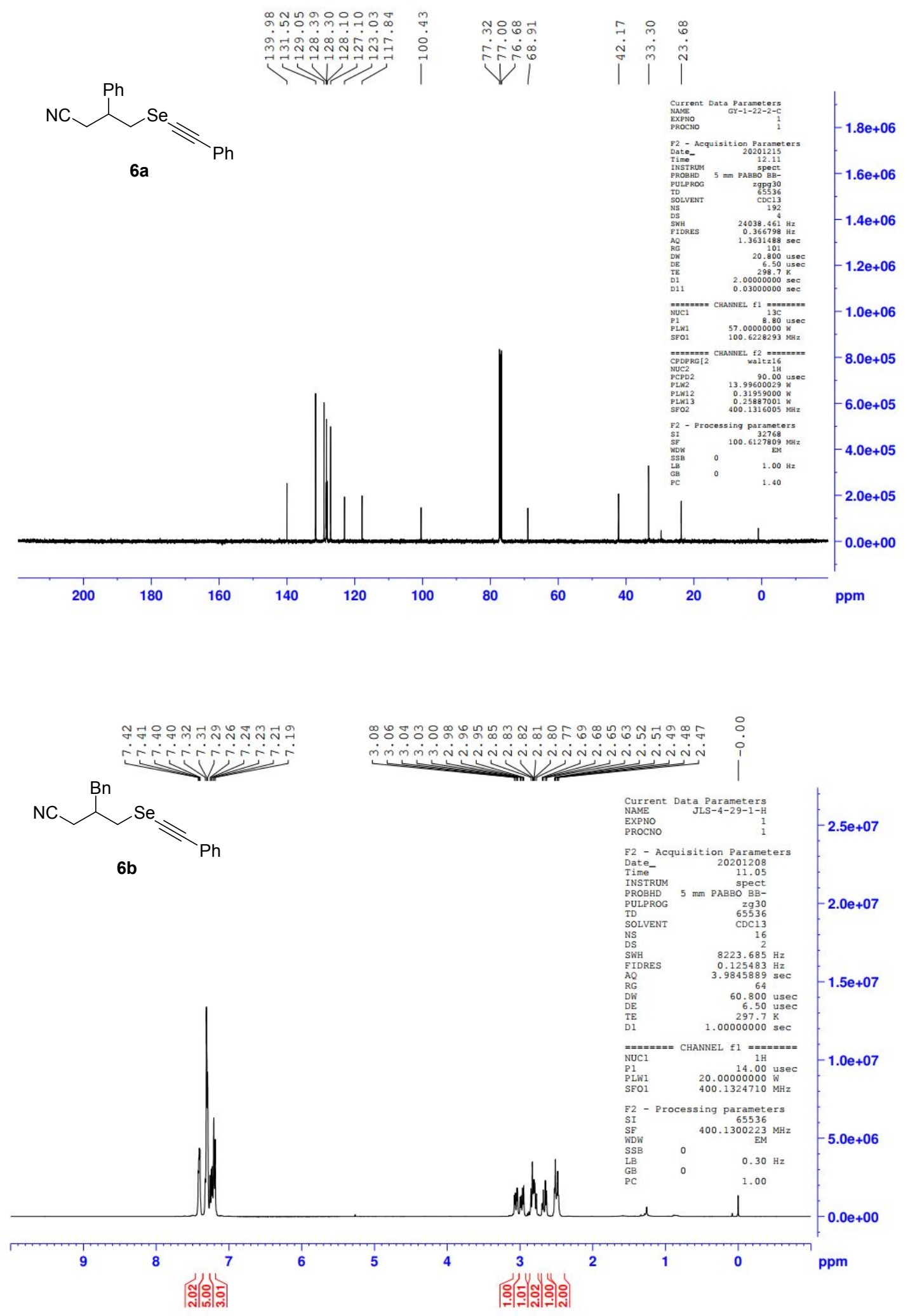

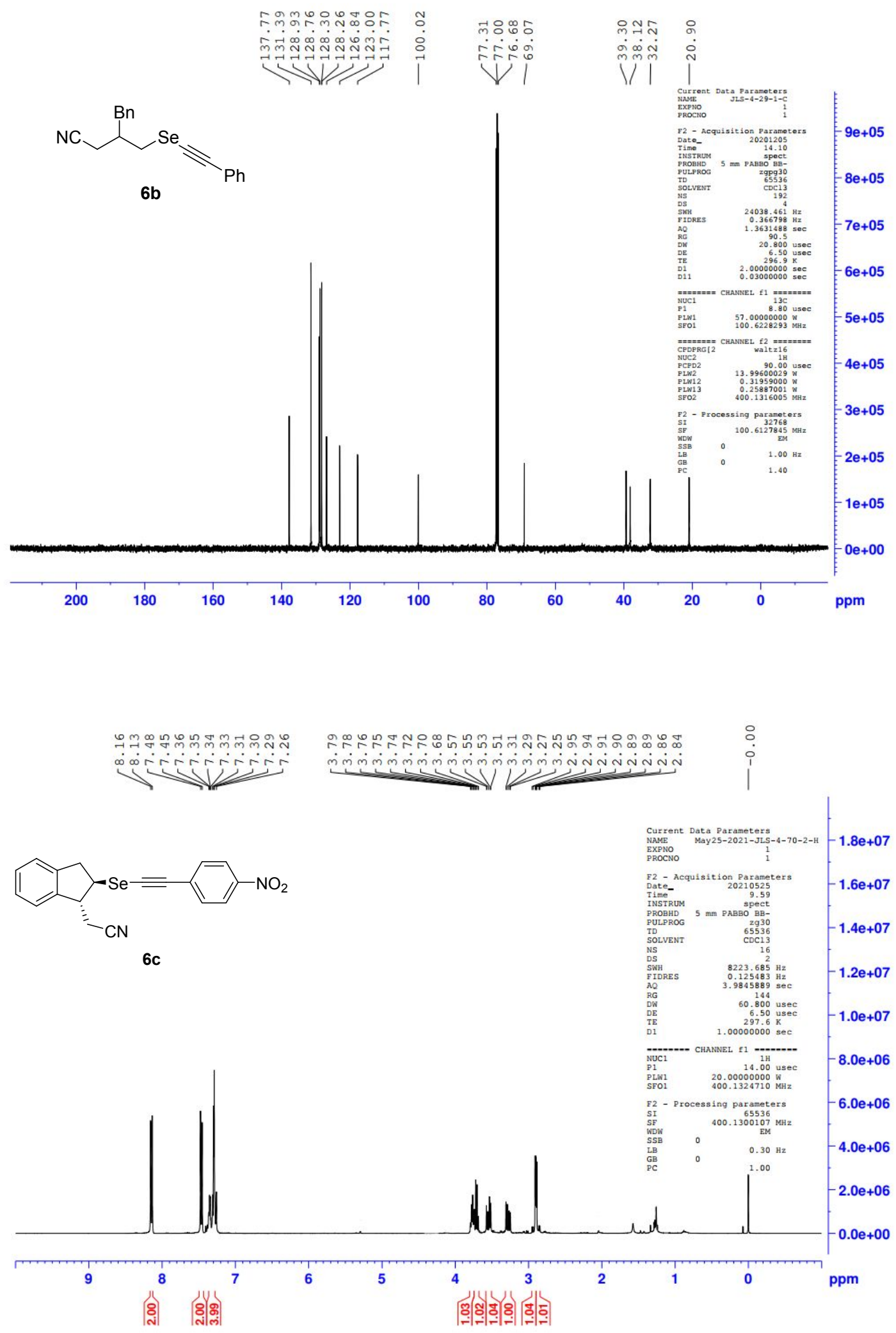


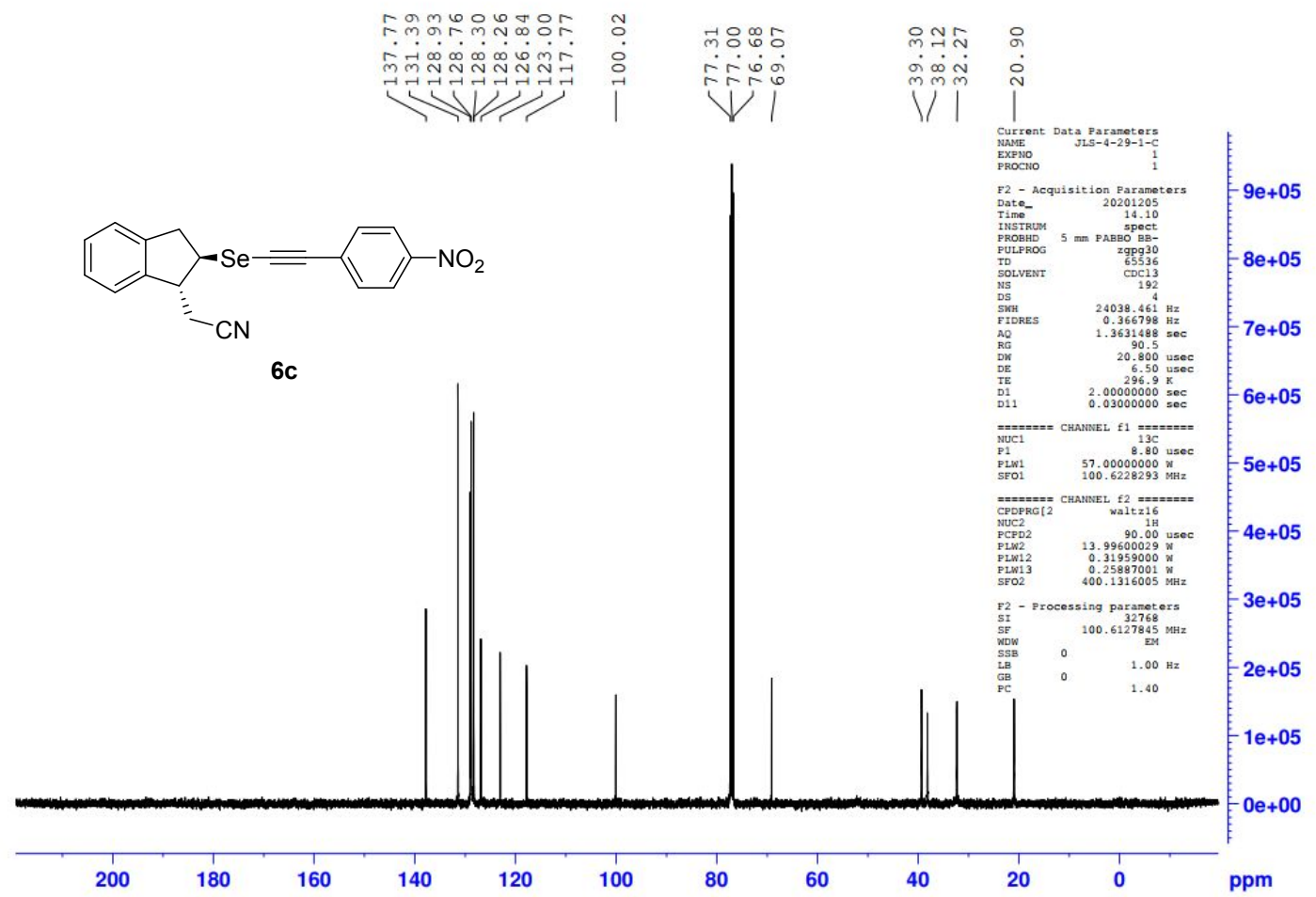<smiles>N#CCCCC=C(c1ccccc1)c1ccccc1</smiles>

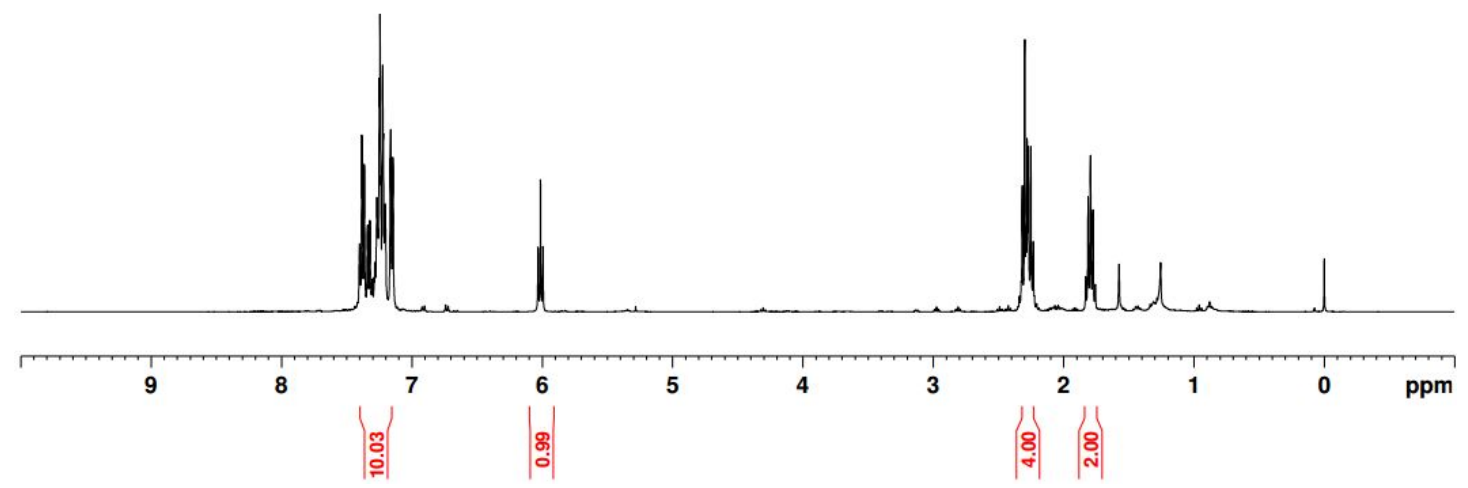




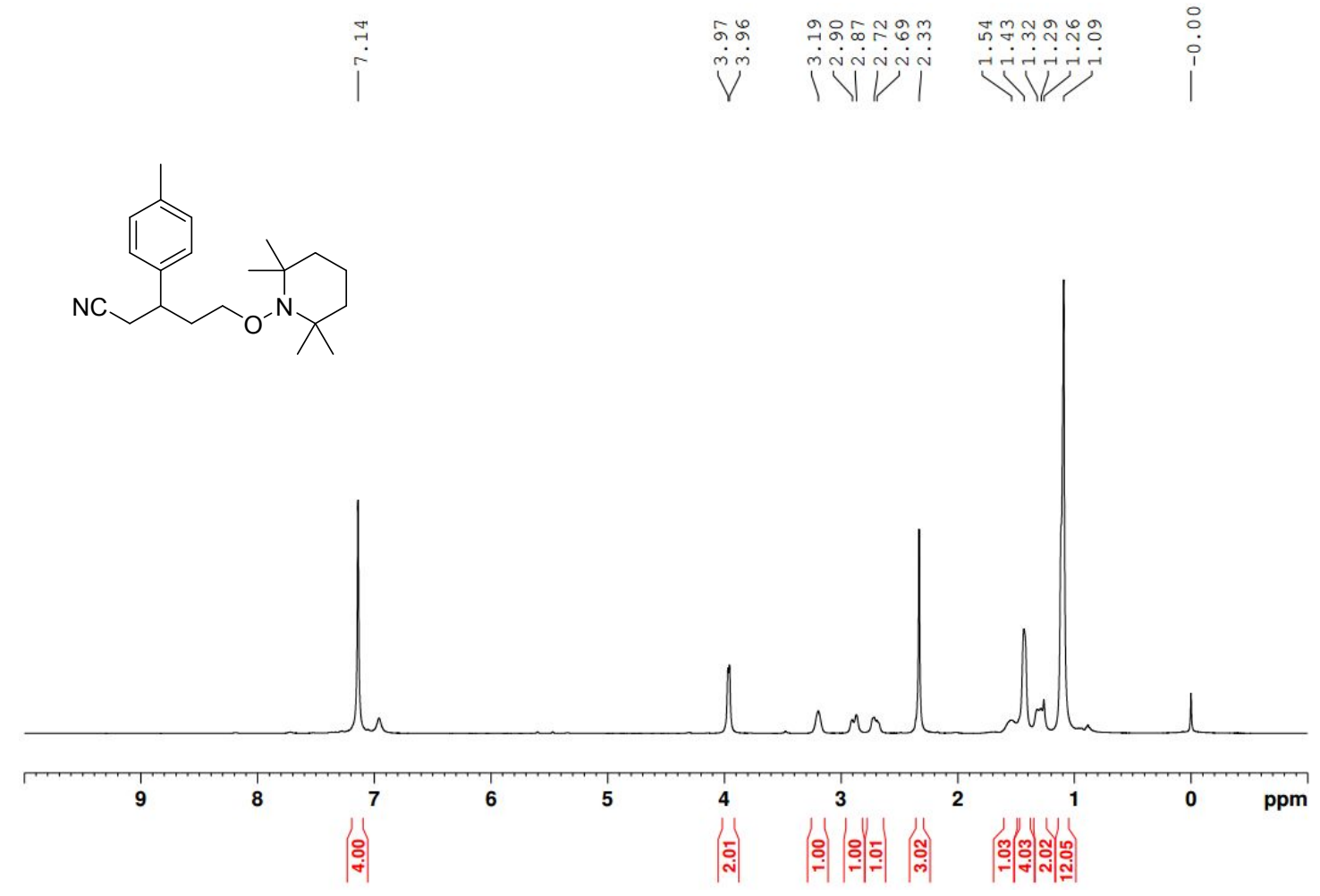




\section{X-ray crystallography structure of compound $6 c$.}

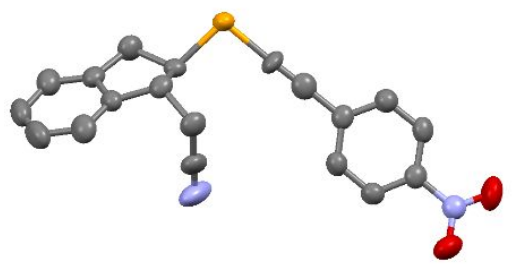

The purified compound $6 \mathrm{c}$ is dissolved in chloroform and $\mathrm{n}$-hexane, and placed in a dark cabinet to slowly evaporate. After several days, a yellow bulk crystal is obtained. The X-ray crystal-structure determinations were obtained on a Bruker Smart CCDC APEX-2 diffractometer at $296(2) \mathrm{K}$.

Table S7. Crystal data and structure refinement for n210524a.

Identification code

Empirical formula

Formula weight

Temperature

Wavelength

Crystal system, space group

Unit cell dimensions

Volume

Z, Calculated density

Absorption coefficient

$\mathrm{F}(000)$

Crystal size

Theta range for data collection

Limiting indices

Reflections collected / unique

Completeness to theta $=25.242$

Absorption correction

Refinement method

Data / restraints / parameters
Semi-empirical from equivalents

Full-matrix least-squares on $\mathrm{F}^{\wedge} 2$

n210524a

C19 H14 N2 O2 Se

381.28

296(2) K

$0.71073 \mathrm{~A}$

Triclinic, $\mathrm{P}-1$

$\mathrm{a}=7.468(4)$ A alpha $=78.319(9)$ deg.

$\mathrm{b}=7.567(4) \mathrm{A} \quad$ beta $=87.888(10) \mathrm{deg}$.

$\mathrm{c}=16.703(8) \mathrm{A}$ gamma $=64.075(8) \mathrm{deg}$.

$829.9(7) \mathrm{A}^{\wedge} 3$

2, $1.526 \mathrm{Mg} / \mathrm{m}^{\wedge} 3$

$2.275 \mathrm{~mm}^{\wedge}-1$

384

$0.220 \times 0.210 \times 0.180 \mathrm{~mm}$

1.247 to $26.499 \mathrm{deg}$.

$-9<=\mathrm{h}<=6,-9<=\mathrm{k}<=9,-20<=\mathrm{k}<=18$

$5160 / 3411[\mathrm{R}(\mathrm{int})=0.0439]$

$3411 / 0 / 217$ 
Goodness-of-fit on $\mathrm{F}^{\wedge} 2$ 1.041

Final $\mathrm{R}$ indices $[\mathrm{I}>2 \operatorname{sigma}(\mathrm{I})] \quad \mathrm{R} 1=0.1019, \mathrm{wR} 2=0.3140$

$\mathrm{R}$ indices (all data) $\mathrm{R} 1=0.1278, \mathrm{wR} 2=0.3260$

Extinction coefficient $\mathrm{n} / \mathrm{a}$

Largest diff. peak and hole 1.444 and -1.417 e. $\mathrm{A}^{\wedge}-3$

Table S8. Atomic coordinates ( x 10^4) and equivalent isotropic displacement parameters $\left(\mathrm{A}^{\wedge} 2 \times 10^{\wedge} 3\right)$ for $\mathrm{N} 210524 \mathrm{~A}$.

$\mathrm{U}(\mathrm{eq})$ is defined as one third of the trace of the orthogonalized

Uij tensor.

\begin{tabular}{|c|c|c|c|c|}
\hline & $\mathrm{x}$ & $\mathrm{y}$ & $\mathrm{z}$ & $\mathrm{U}(\mathrm{eq})$ \\
\hline $\operatorname{Se}(1)$ & $1982(2)$ & $4108(2)$ & $3058(1)$ & $49(1)$ \\
\hline $\mathrm{O}(1)$ & 2399(17) & 10161(18) & $7337(6)$ & $82(3)$ \\
\hline $\mathrm{O}(2)$ & $3177(16)$ & $12004(15)$ & $6406(6)$ & $76(3)$ \\
\hline $\mathrm{N}(1)$ & $2520(20)$ & 10690(19) & $2084(10)$ & $97(5)$ \\
\hline $\mathrm{N}(2)$ & $2690(13)$ & $10644(14)$ & $6631(6)$ & $50(2)$ \\
\hline $\mathrm{C}(1)$ & $3540(20)$ & $9036(19)$ & $2125(8)$ & $62(3)$ \\
\hline$C(2)$ & $4763(17)$ & $6896(18)$ & $2201(8)$ & $55(3)$ \\
\hline$C(3)$ & $3799(15)$ & $5927(16)$ & $1748(7)$ & $47(2)$ \\
\hline$C(4)$ & $3306(16)$ & $6813(15)$ & $841(6)$ & $46(2)$ \\
\hline$C(5)$ & $4290(20)$ & $7510(20)$ & $258(8)$ & $64(3)$ \\
\hline$C(6)$ & $3550(30)$ & $8180(20)$ & $-561(8)$ & $77(4)$ \\
\hline$C(7)$ & $1840(20)$ & $8140(20)$ & $-772(8)$ & $69(4)$ \\
\hline $\mathrm{C}(8)$ & $820(20)$ & $7379(19)$ & $-187(8)$ & $65(3)$ \\
\hline$C(9)$ & $1536(17)$ & $6742(15)$ & $613(7)$ & $49(2)$ \\
\hline$C(10)$ & $686(18)$ & $5930(20)$ & $1349(7)$ & $57(3)$ \\
\hline$C(11)$ & $1805(15)$ & $6104(14)$ & $2073(6)$ & $41(2)$ \\
\hline$C(12)$ & $2136(18)$ & $5541(16)$ & $3813(7)$ & $52(3)$ \\
\hline$C(13)$ & $2268(16)$ & 6359(19) & $4306(8)$ & $55(3)$ \\
\hline$C(14)$ & $2367(14)$ & $7456(16)$ & $4905(6)$ & $44(2)$ \\
\hline$C(15)$ & $2167(17)$ & $6823(18)$ & $5723(7)$ & $52(3)$ \\
\hline$C(16)$ & $2243(15)$ & 7861(17) & $6294(6)$ & $49(2)$ \\
\hline
\end{tabular}


$\mathrm{C}(17) \quad 2551(14) \quad 9558(16) \quad 6036(6) \quad 46(2)$

$\begin{array}{lllll}C(18) & 2730(15) & 10243(16) & 5224(7) & 47(2)\end{array}$

$\mathrm{C}(19) \quad 2648(16) \quad 9182(17) \quad 4671(6) \quad 47(2)$ 
Table S9. Bond lengths [A] and angles [deg] for N210524A.

\begin{tabular}{|c|c|}
\hline $\operatorname{Se}(1)-C(12)$ & $1.857(10)$ \\
\hline $\operatorname{Se}(1)-C(11)$ & $1.960(10)$ \\
\hline $\mathrm{O}(1)-\mathrm{N}(2)$ & $1.203(13)$ \\
\hline $\mathrm{O}(2)-\mathrm{N}(2)$ & $1.222(13)$ \\
\hline $\mathrm{N}(1)-\mathrm{C}(1)$ & $1.134(18)$ \\
\hline $\mathrm{N}(2)-\mathrm{C}(17)$ & $1.444(14)$ \\
\hline $\mathrm{C}(1)-\mathrm{C}(2)$ & $1.451(18)$ \\
\hline$C(2)-C(3)$ & $1.533(15)$ \\
\hline $\mathrm{C}(2)-\mathrm{H}(2 \mathrm{~A})$ & 0.9700 \\
\hline $\mathrm{C}(2)-\mathrm{H}(2 \mathrm{~B})$ & 0.9700 \\
\hline$C(3)-C(4)$ & $1.516(15)$ \\
\hline $\mathrm{C}(3)-\mathrm{C}(11)$ & $1.525(15)$ \\
\hline $\mathrm{C}(3)-\mathrm{H}(3)$ & 0.9800 \\
\hline$C(4)-C(5)$ & $1.360(17)$ \\
\hline$C(4)-C(9)$ & $1.414(16)$ \\
\hline$C(5)-C(6)$ & $1.40(2)$ \\
\hline $\mathrm{C}(5)-\mathrm{H}(5)$ & 0.9300 \\
\hline$C(6)-C(7)$ & $1.35(2)$ \\
\hline $\mathrm{C}(6)-\mathrm{H}(6)$ & 0.9300 \\
\hline$C(7)-C(8)$ & $1.40(2)$ \\
\hline $\mathrm{C}(7)-\mathrm{H}(7)$ & 0.9300 \\
\hline $\mathrm{C}(8)-\mathrm{C}(9)$ & $1.368(17)$ \\
\hline $\mathrm{C}(8)-\mathrm{H}(8)$ & 0.9300 \\
\hline $\mathrm{C}(9)-\mathrm{C}(10)$ & $1.507(17)$ \\
\hline$C(10)-C(11)$ & $1.556(15)$ \\
\hline $\mathrm{C}(10)-\mathrm{H}(10 \mathrm{~A})$ & 0.9700 \\
\hline $\mathrm{C}(10)-\mathrm{H}(10 \mathrm{~B})$ & 0.9700 \\
\hline $\mathrm{C}(11)-\mathrm{H}(11)$ & 0.9800 \\
\hline$C(12)-C(13)$ & $1.156(16)$ \\
\hline
\end{tabular}




\begin{tabular}{|c|c|}
\hline$C(13)-C(14)$ & $1.446(16)$ \\
\hline$C(14)-C(15)$ & $1.379(16)$ \\
\hline$C(14)-C(19)$ & $1.389(16)$ \\
\hline$C(15)-C(16)$ & $1.370(16)$ \\
\hline $\mathrm{C}(15)-\mathrm{H}(15)$ & 0.9300 \\
\hline$C(16)-C(17)$ & $1.384(16)$ \\
\hline $\mathrm{C}(16)-\mathrm{H}(16)$ & 0.9300 \\
\hline $\mathrm{C}(17)-\mathrm{C}(18)$ & $1.374(15)$ \\
\hline$C(18)-C(19)$ & $1.359(15)$ \\
\hline $\mathrm{C}(18)-\mathrm{H}(18)$ & 0.9300 \\
\hline C(19)-H(19) & 0.9300 \\
\hline $\mathrm{C}(12)-\mathrm{Se}(1)-\mathrm{C}(11)$ & $96.7(5)$ \\
\hline $\mathrm{O}(1)-\mathrm{N}(2)-\mathrm{O}(2)$ & $121.2(11)$ \\
\hline $\mathrm{O}(1)-\mathrm{N}(2)-\mathrm{C}(17)$ & $120.2(10)$ \\
\hline $\mathrm{O}(2)-\mathrm{N}(2)-\mathrm{C}(17)$ & $118.5(10)$ \\
\hline $\mathrm{N}(1)-\mathrm{C}(1)-\mathrm{C}(2)$ & $176.9(17)$ \\
\hline$C(1)-C(2)-C(3)$ & $112.5(9)$ \\
\hline $\mathrm{C}(1)-\mathrm{C}(2)-\mathrm{H}(2 \mathrm{~A})$ & 109.1 \\
\hline $\mathrm{C}(3)-\mathrm{C}(2)-\mathrm{H}(2 \mathrm{~A})$ & 109.1 \\
\hline $\mathrm{C}(1)-\mathrm{C}(2)-\mathrm{H}(2 \mathrm{~B})$ & 109.1 \\
\hline $\mathrm{C}(3)-\mathrm{C}(2)-\mathrm{H}(2 \mathrm{~B})$ & 109.1 \\
\hline $\mathrm{H}(2 \mathrm{~A})-\mathrm{C}(2)-\mathrm{H}(2 \mathrm{~B})$ & 107.8 \\
\hline$C(4)-C(3)-C(11)$ & $103.3(8)$ \\
\hline$C(4)-C(3)-C(2)$ & $115.5(10)$ \\
\hline$C(11)-C(3)-C(2)$ & $113.3(9)$ \\
\hline $\mathrm{C}(4)-\mathrm{C}(3)-\mathrm{H}(3)$ & 108.1 \\
\hline $\mathrm{C}(11)-\mathrm{C}(3)-\mathrm{H}(3)$ & 108.1 \\
\hline $\mathrm{C}(2)-\mathrm{C}(3)-\mathrm{H}(3)$ & 108.1 \\
\hline$C(5)-C(4)-C(9)$ & $119.5(11)$ \\
\hline$C(5)-C(4)-C(3)$ & $131.6(11)$ \\
\hline$C(9)-C(4)-C(3)$ & $108.8(9)$ \\
\hline
\end{tabular}




\begin{tabular}{|c|c|}
\hline$C(4)-C(5)-C(6)$ & $120.4(14)$ \\
\hline $\mathrm{C}(4)-\mathrm{C}(5)-\mathrm{H}(5)$ & 119.8 \\
\hline $\mathrm{C}(6)-\mathrm{C}(5)-\mathrm{H}(5)$ & 119.8 \\
\hline$C(7)-C(6)-C(5)$ & $119.8(13)$ \\
\hline $\mathrm{C}(7)-\mathrm{C}(6)-\mathrm{H}(6)$ & 120.1 \\
\hline $\mathrm{C}(5)-\mathrm{C}(6)-\mathrm{H}(6)$ & 120.1 \\
\hline$C(6)-C(7)-C(8)$ & $121.0(12)$ \\
\hline $\mathrm{C}(6)-\mathrm{C}(7)-\mathrm{H}(7)$ & 119.5 \\
\hline $\mathrm{C}(8)-\mathrm{C}(7)-\mathrm{H}(7)$ & 119.5 \\
\hline $\mathrm{C}(9)-\mathrm{C}(8)-\mathrm{C}(7)$ & $119.0(13)$ \\
\hline $\mathrm{C}(9)-\mathrm{C}(8)-\mathrm{H}(8)$ & 120.5 \\
\hline $\mathrm{C}(7)-\mathrm{C}(8)-\mathrm{H}(8)$ & 120.5 \\
\hline $\mathrm{C}(8)-\mathrm{C}(9)-\mathrm{C}(4)$ & $120.3(11)$ \\
\hline $\mathrm{C}(8)-\mathrm{C}(9)-\mathrm{C}(10)$ & $128.8(11)$ \\
\hline$C(4)-C(9)-C(10)$ & $110.9(9)$ \\
\hline$C(9)-C(10)-C(11)$ & $102.3(9)$ \\
\hline $\mathrm{C}(9)-\mathrm{C}(10)-\mathrm{H}(10 \mathrm{~A})$ & 111.3 \\
\hline $\mathrm{C}(11)-\mathrm{C}(10)-\mathrm{H}(10 \mathrm{~A})$ & 111.3 \\
\hline $\mathrm{C}(9)-\mathrm{C}(10)-\mathrm{H}(10 \mathrm{~B})$ & 111.3 \\
\hline $\mathrm{C}(11)-\mathrm{C}(10)-\mathrm{H}(10 \mathrm{~B})$ & 111.3 \\
\hline $\mathrm{H}(10 \mathrm{~A})-\mathrm{C}(10)-\mathrm{H}(10 \mathrm{~B})$ & 109.2 \\
\hline$C(3)-C(11)-C(10)$ & $104.9(9)$ \\
\hline $\mathrm{C}(3)-\mathrm{C}(11)-\mathrm{Se}(1)$ & $115.3(7)$ \\
\hline$C(10)-C(11)-S e(1)$ & $110.8(7)$ \\
\hline $\mathrm{C}(3)-\mathrm{C}(11)-\mathrm{H}(11)$ & 108.5 \\
\hline $\mathrm{C}(10)-\mathrm{C}(11)-\mathrm{H}(11)$ & 108.5 \\
\hline $\mathrm{Se}(1)-\mathrm{C}(11)-\mathrm{H}(11)$ & 108.5 \\
\hline $\mathrm{C}(13)-\mathrm{C}(12)-\mathrm{Se}(1)$ & $177.2(11)$ \\
\hline$C(12)-C(13)-C(14)$ & $177.6(13)$ \\
\hline$C(15)-C(14)-C(19)$ & $118.3(10)$ \\
\hline$C(15)-C(14)-C(13)$ & $120.7(11)$ \\
\hline $\mathrm{C}(19)-\mathrm{C}(14)-\mathrm{C}(13)$ & $121.0(10)$ \\
\hline
\end{tabular}




$\begin{array}{ll}\mathrm{C}(16)-\mathrm{C}(15)-\mathrm{C}(14) & 121.2(11) \\ \mathrm{C}(16)-\mathrm{C}(15)-\mathrm{H}(15) & 119.4 \\ \mathrm{C}(14)-\mathrm{C}(15)-\mathrm{H}(15) & 119.4 \\ \mathrm{C}(15)-\mathrm{C}(16)-\mathrm{C}(17) & 118.6(10) \\ \mathrm{C}(15)-\mathrm{C}(16)-\mathrm{H}(16) & 120.7 \\ \mathrm{C}(17)-\mathrm{C}(16)-\mathrm{H}(16) & 120.7 \\ \mathrm{C}(18)-\mathrm{C}(17)-\mathrm{C}(16) & 121.6(10) \\ \mathrm{C}(18)-\mathrm{C}(17)-\mathrm{N}(2) & 118.9(10) \\ \mathrm{C}(16)-\mathrm{C}(17)-\mathrm{N}(2) & 119.5(10) \\ \mathrm{C}(19)-\mathrm{C}(18)-\mathrm{C}(17) & 118.4(10) \\ \mathrm{C}(19)-\mathrm{C}(18)-\mathrm{H}(18) & 120.8 \\ \mathrm{C}(17)-\mathrm{C}(18)-\mathrm{H}(18) & 120.8 \\ \mathrm{C}(18)-\mathrm{C}(19)-\mathrm{C}(14) & 121.9(10) \\ \mathrm{C}(18)-\mathrm{C}(19)-\mathrm{H}(19) & 119.1 \\ \mathrm{C}(14)-\mathrm{C}(19)-\mathrm{H}(19) & 119.1\end{array}$

Symmetry transformations used to generate equivalent atoms: 
Table S10. Anisotropic displacement parameters ( $\left.\mathrm{A}^{\wedge} 2 \times 10^{\wedge} 3\right)$ for N210524A.

The anisotropic displacement factor exponent takes the form:

$-2 \mathrm{pi}^{\wedge} 2\left[\mathrm{~h}^{\wedge} 2 \mathrm{a}^{* \wedge} 2 \mathrm{U} 11+\ldots+2 \mathrm{hk} \mathrm{a} \mathrm{a}^{*} \mathrm{U} 12\right]$

\begin{tabular}{lllllll}
\hline \multicolumn{1}{c}{$\mathrm{U} 11$} & $\mathrm{U} 22$ & $\mathrm{U} 33$ & $\mathrm{U} 23$ & $\mathrm{U} 13$ & $\mathrm{U} 12$ \\
\hline $\mathrm{Se}(1)$ & $70(1)$ & $43(1)$ & $45(1)$ & $-10(1)$ & $4(1)$ & $-33(1)$ \\
$\mathrm{O}(1)$ & $94(7)$ & $103(8)$ & $50(5)$ & $-30(5)$ & $5(5)$ & $-38(6)$ \\
$\mathrm{O}(2)$ & $93(7)$ & $62(5)$ & $84(6)$ & $-23(5)$ & $-13(5)$ & $-39(5)$ \\
$\mathrm{N}(1)$ & $125(12)$ & $55(7)$ & $111(11)$ & $-24(7)$ & $-43(9)$ & $-34(8)$ \\
$\mathrm{N}(2)$ & $41(5)$ & $48(5)$ & $54(6)$ & $-10(4)$ & $0(4)$ & $-14(4)$ \\
$\mathrm{C}(1)$ & $77(8)$ & $55(7)$ & $69(8)$ & $-14(6)$ & $-11(6)$ & $-41(7)$ \\
$\mathrm{C}(2)$ & $44(6)$ & $59(7)$ & $65(7)$ & $-16(5)$ & $-5(5)$ & $-24(5)$ \\
$\mathrm{C}(3)$ & $39(5)$ & $42(5)$ & $55(6)$ & $-17(5)$ & $0(4)$ & $-10(4)$ \\
$\mathrm{C}(4)$ & $53(6)$ & $40(5)$ & $43(5)$ & $-11(4)$ & $8(4)$ & $-19(5)$ \\
$\mathrm{C}(5)$ & $58(7)$ & $66(8)$ & $60(7)$ & $-20(6)$ & $14(6)$ & $-18(6)$ \\
$\mathrm{C}(6)$ & $103(12)$ & $68(9)$ & $53(7)$ & $-10(6)$ & $25(7)$ & $-34(8)$ \\
$\mathrm{C}(7)$ & $96(11)$ & $58(7)$ & $40(6)$ & $-10(5)$ & $7(6)$ & $-23(7)$ \\
$\mathrm{C}(8)$ & $72(8)$ & $56(7)$ & $56(7)$ & $-13(6)$ & $-1(6)$ & $-18(6)$ \\
$\mathrm{C}(9)$ & $58(6)$ & $37(5)$ & $51(6)$ & $-14(4)$ & $-1(5)$ & $-17(5)$ \\
$\mathrm{C}(10)$ & $51(6)$ & $62(7)$ & $59(7)$ & $-12(5)$ & $-6(5)$ & $-26(6)$ \\
$\mathrm{C}(11)$ & $41(5)$ & $31(4)$ & $49(5)$ & $-6(4)$ & $0(4)$ & $-15(4)$ \\
$\mathrm{C}(12)$ & $65(7)$ & $44(6)$ & $49(6)$ & $-23(5)$ & $-3(5)$ & $-21(5)$ \\
$\mathrm{C}(13)$ & $37(5)$ & $62(7)$ & $61(7)$ & $-9(6)$ & $-1(5)$ & $-18(5)$ \\
$\mathrm{C}(14)$ & $31(5)$ & $48(5)$ & $48(5)$ & $-13(4)$ & $0(4)$ & $-11(4)$ \\
$\mathrm{C}(15)$ & $54(6)$ & $51(6)$ & $52(6)$ & $-10(5)$ & $2(5)$ & $-23(5)$ \\
$\mathrm{C}(16)$ & $41(5)$ & $57(6)$ & $41(5)$ & $-7(5)$ & $-4(4)$ & $-16(5)$ \\
$\mathrm{C}(17)$ & $32(5)$ & $48(6)$ & $49(6)$ & $-9(4)$ & $-5(4)$ & $-11(4)$ \\
$\mathrm{C}(18)$ & $44(5)$ & $48(6)$ & $51(6)$ & $-8(5)$ & $4(4)$ & $-23(5)$ \\
$\mathrm{C}(19)$ & $47(6)$ & $56(6)$ & $42(5)$ & $-8(4)$ & $3(4)$ & $-25(5)$ \\
\hline & & & & & & \\
\hline
\end{tabular}


Table S11. Hydrogen coordinates ( x 10^4) and isotropic

displacement parameters $\left(\mathrm{A}^{\wedge} 2 \times 10^{\wedge} 3\right)$ for $\mathrm{N} 210524 \mathrm{~A}$.

\begin{tabular}{lcccc}
\hline & \multicolumn{3}{c}{ y } & U(eq) \\
\hline $\mathrm{H}(2 \mathrm{~A})$ & 4990 & 6249 & 2776 & 66 \\
$\mathrm{H}(2 \mathrm{~B})$ & 6048 & 6676 & 1982 & 66 \\
$\mathrm{H}(3)$ & 4713 & 4497 & 1815 & 57 \\
$\mathrm{H}(5)$ & 5470 & 7534 & 403 & 77 \\
$\mathrm{H}(6)$ & 4234 & 8652 & -958 & 92 \\
$\mathrm{H}(7)$ & 1328 & 8629 & -1313 & 82 \\
$\mathrm{H}(8)$ & -329 & 7307 & -340 & 78 \\
$\mathrm{H}(10 \mathrm{~A})$ & 965 & 4539 & 1366 & 68 \\
$\mathrm{H}(10 \mathrm{~B})$ & -743 & 6723 & 1351 & 68 \\
$\mathrm{H}(11)$ & 1058 & 7447 & 2192 & 49 \\
$\mathrm{H}(15)$ & 1976 & 5671 & 5890 & 63 \\
$\mathrm{H}(16)$ & 2090 & 7435 & 6844 & 58 \\
$\mathrm{H}(18)$ & 2904 & 11404 & 5056 & 56 \\
$\mathrm{H}(19)$ & 2783 & 9625 & 4121 & 57 \\
& & & & \\
\hline
\end{tabular}


Table S12. Torsion angles [deg] for N210524A.

\begin{tabular}{lc}
\hline$C(1)-C(2)-C(3)-C(4)$ & $57.5(14)$ \\
$C(1)-C(2)-C(3)-C(11)$ & $-61.4(14)$ \\
$C(11)-C(3)-C(4)-C(5)$ & $162.4(12)$ \\
$C(2)-C(3)-C(4)-C(5)$ & $38.2(17)$ \\
$C(11)-C(3)-C(4)-C(9)$ & $-21.3(11)$ \\
$C(2)-C(3)-C(4)-C(9)$ & $-145.5(9)$ \\
$C(9)-C(4)-C(5)-C(6)$ & $1.1(18)$ \\
$C(3)-C(4)-C(5)-C(6)$ & $177.1(12)$ \\
$C(4)-C(5)-C(6)-C(7)$ & $0(2)$ \\
$C(5)-C(6)-C(7)-C(8)$ & $-2(2)$ \\
$C(6)-C(7)-C(8)-C(9)$ & $3(2)$ \\
$C(7)-C(8)-C(9)-C(4)$ & $-1.4(17)$ \\
$C(7)-C(8)-C(9)-C(10)$ & $178.2(12)$ \\
$C(5)-C(4)-C(9)-C(8)$ & $-0.4(16)$ \\
$C(3)-C(4)-C(9)-C(8)$ & $-177.2(10)$ \\
$C(5)-C(4)-C(9)-C(10)$ & $179.9(11)$ \\
$C(3)-C(4)-C(9)-C(10)$ & $3.1(12)$ \\
$C(8)-C(9)-C(10)-C(11)$ & $-163.7(11)$ \\
$C(4)-C(9)-C(10)-C(11)$ & $16.0(12)$ \\
$C(4)-C(3)-C(11)-C(10)$ & $30.5(10)$ \\
$C(2)-C(3)-C(11)-C(10)$ & $156.2(9)$ \\
$C(4)-C(3)-C(11)-S e(1)$ & $152.7(7)$ \\
$C(2)-C(3)-C(11)-S e(1)$ & $-81.6(10)$ \\
$C(9)-C(10)-C(11)-C(3)$ & $-28.6(11)$ \\
$C(9)-C(10)-C(11)-S e(1)$ & $-153.6(7)$ \\
$C(19)-C(14)-C(15)-C(16)$ & $-C(14)-C(15)-C(16)$ \\
$C(15)-C(16)-C(17)$ & $-C(17)-C(18)$ \\
$C(16)$
\end{tabular}




$\begin{array}{lc}\mathrm{C}(15)-\mathrm{C}(16)-\mathrm{C}(17)-\mathrm{N}(2) & -178.4(9) \\ \mathrm{O}(1)-\mathrm{N}(2)-\mathrm{C}(17)-\mathrm{C}(18) & 174.8(10) \\ \mathrm{O}(2)-\mathrm{N}(2)-\mathrm{C}(17)-\mathrm{C}(18) & -7.9(14) \\ \mathrm{O}(1)-\mathrm{N}(2)-\mathrm{C}(17)-\mathrm{C}(16) & -5.2(15) \\ \mathrm{O}(2)-\mathrm{N}(2)-\mathrm{C}(17)-\mathrm{C}(16) & 172.1(10) \\ \mathrm{C}(16)-\mathrm{C}(17)-\mathrm{C}(18)-\mathrm{C}(19) & -1.6(15) \\ \mathrm{N}(2)-\mathrm{C}(17)-\mathrm{C}(18)-\mathrm{C}(19) & 178.4(9) \\ \mathrm{C}(17)-\mathrm{C}(18)-\mathrm{C}(19)-\mathrm{C}(14) & 0.7(16) \\ \mathrm{C}(15)-\mathrm{C}(14)-\mathrm{C}(19)-\mathrm{C}(18) & 0.1(16) \\ \mathrm{C}(13)-\mathrm{C}(14)-\mathrm{C}(19)-\mathrm{C}(18) & 179.5(10)\end{array}$

Symmetry transformations used to generate equivalent atoms:

Table S13. Hydrogen bonds for N210524A [A and deg.].

$\begin{array}{lllll}\text { D-H...A } & \text { d(D-H) } & \text { d(H...A) } & \text { d(D...A }) & <(\text { DHA })\end{array}$ 